\title{
Heisenberg-Lie commutation relations in Banach algebras
}

\author{
Niels Jakob Laustsen and Sergei D. Silvestrov
}

\begin{abstract}
Given $q_{1}, q_{2} \in \mathbb{C} \backslash\{0\}$, we construct a unital Banach algebra $\mathscr{B}_{q_{1}, q_{2}}$ which contains a universal normalized solution to the $\left(q_{1}, q_{2}\right)$-deformed Heisenberg-Lie commutation relations in the following specific sense: (i) $\mathscr{B}_{q_{1}, q_{2}}$ contains elements $b_{1}, b_{2}$, and $b_{3}$ which satisfy the $\left(q_{1}, q_{2}\right)$-deformed Heisenberg-Lie commutation relations (that is, $b_{1} b_{2}-q_{1} b_{2} b_{1}=b_{3}, q_{2} b_{1} b_{3}-b_{3} b_{1}=0$, and $\left.b_{2} b_{3}-q_{2} b_{3} b_{2}=0\right)$, and $\left\|b_{1}\right\|=\left\|b_{2}\right\|=1$; (ii) whenever a unital Banach algebra $\mathscr{A}$ contains elements $a_{1}, a_{2}$, and $a_{3}$ satisfying the $\left(q_{1}, q_{2}\right)$-deformed Heisenberg-Lie commutation relations and $\left\|a_{1}\right\|,\left\|a_{2}\right\| \leqslant 1$, there is a unique bounded unital algebra homomorphism $\varphi: \mathscr{B}_{q_{1}, q_{2}} \rightarrow \mathscr{A}$ such that $\varphi\left(b_{j}\right)=a_{j}$ for $j=1,2,3$.

For $q_{1}, q_{2} \in \mathbb{R} \backslash\{0\}$, we obtain a counterpart of the above result for Banach $*$-algebras. In contrast, we show that if $q_{1}, q_{2} \in(-\infty, 0), q_{1}, q_{2} \in(0,1)$, or $q_{1}, q_{2} \in(1, \infty)$, then a $C^{*}$-algebra cannot contain a non-zero solution to the $*$-algebraic counterpart of the $\left(q_{1}, q_{2}\right)$-deformed Heisenberg-Lie commutation relations. However, for many other pairs $q_{1}, q_{2} \in \mathbb{R} \backslash\{0\}$, an explicit construction based on a weighted shift operator on $\ell_{2}(\mathbb{Z})$ produces a non-zero solution to the $*$-algebraic counterpart of the $\left(q_{1}, q_{2}\right)$-deformed Heisenberg-Lie commutation relations; we determine all such pairs.

2000 Mathematics Subject Classification: primary 46H15, 46K05; secondary 47B37, 47B47, 43A20.

Key words and phrases: Heisenberg-Lie commutation relations, Banach algebra, Banach $*$-algebra, $C^{*}$-algebra, weighted shift operator.
\end{abstract}

\section{Introduction and main results}

Let $q_{1}$ and $q_{2}$ be non-zero complex numbers. We say that three elements $b_{1}, b_{2}$, and $b_{3}$ of a complex algebra satisfy the $\left(q_{1}, q_{2}\right)$-deformed Heisenberg-Lie commutation relations if

$$
b_{1} b_{2}-q_{1} b_{2} b_{1}=b_{3}, \quad q_{2} b_{1} b_{3}-b_{3} b_{1}=0, \quad \text { and } \quad b_{2} b_{3}-q_{2} b_{3} b_{2}=0 .
$$

In the case where $q_{1}=q_{2}=1$, these relations reduce to the classical Heisenberg-Lie commutation relations, while for $q_{1}=q_{2}=-1$, they are known as the coloured HeisenbergLie commutation relations (see $[23,25])$. 
After presenting the paper [23] at the Second Øresund Symposium in Noncommutative Analysis and Geometry, held in Copenhagen 2003, the second author raised the problem of how to realize the $\left(q_{1}, q_{2}\right)$-deformed Heisenberg-Lie commutation relations inside a normed algebra. It is not hard to see that non-zero realizations exist; for instance, we have the following example.

1.1 Example. The three complex $(3 \times 3)$-matrices

$$
b_{1}=\left(\begin{array}{ccc}
0 & 0 & 0 \\
0 & 0 & 0 \\
0 & 1 & 0
\end{array}\right), \quad b_{2}=\left(\begin{array}{ccc}
0 & 0 & 0 \\
1 & 0 & 0 \\
0 & 0 & 0
\end{array}\right), \quad \text { and } \quad b_{3}=\left(\begin{array}{ccc}
0 & 0 & 0 \\
0 & 0 & 0 \\
1 & 0 & 0
\end{array}\right)
$$

satisfy $b_{1} b_{2}=b_{3}$ and $b_{j} b_{k}=0$ for $(j, k) \neq(1,2)$, and therefore (1.1) is trivially satisfied in this case, no matter what $q_{1}$ and $q_{2}$ are.

Whether or not a given set of commutation relations can be realized inside a normed algebra is an important question with deep connections to the structure theory of algebras and their representations. The seminal result in this area is the Wintner-Wielandt theorem (see $[27,28]$, or $[16,18]$ for more modern accounts) stating that no unital normed algebra $\mathscr{A}$ contains elements $a$ and $b$ satisfying Heisenberg's canonical commutation relation

$$
a b-b a=1_{\mathscr{A}},
$$

where $1_{\mathscr{A}}$ denotes the identity of $\mathscr{A}$. The unital complex algebra with two generators $a$ and $b$ and defining commutation relation (1.2) is called the Heisenberg algebra; it is also known as the Weyl algebra, especially in the algebra literature. The Wintner-Wielandt theorem implies that this algebra cannot be normed.

The motivation behind this result comes from Quantum Physics. Indeed, it was prompted by Heisenberg's fundamental postulate that, up to a constant, the operators representing the quantum-mechanical momentum and position, respectively, satisfy the commutation relation (1.2). The Wintner-Wielandt theorem implies that these operators cannot both be bounded. Thus any mathematical formulation of Quantum Mechanics in terms of operators acting on a Hilbert (or a Banach) space must necessarily involve unbounded operators.

Heisenberg's canonical commutation relation (1.2) is closely related to three-dimensional Lie algebras and the commutation relations (1.1); indeed, it arises from (1.1) by taking $q_{1}=q_{2}=1, b_{1}=a, b_{2}=b$, and $b_{3}=1_{\mathscr{A}}$ (the identity of the underlying algebra).

If instead we take $q_{1}=q_{2}=-1$, then the algebra with generators $b_{1}, b_{2}$, and $b_{3}$ satisfying the relations (1.1) is known as the universal envelope of the coloured HeisenbergLie algebra. This is one of the main examples of a non-commutative, three-dimensional coloured Lie algebra; a detailed investigation of this class of algebras can be found in [25]. Coloured Lie algebras generalize Lie algebras and Lie superalgebras by allowing a grading by an arbitrary abelian group and by twisting the skew-symmetry and Jacobi identity by a commutation factor. They were originally introduced in Theoretical Physics in the 1970s, 
motivated by problems in particle physics, field theory, models of gravity and string theory (see $[10,17]$ ), but they have subsequently taken on a life of their own in Mathematics (see for instance $[1-3,5,11-15,20-25])$.

Operator representations of coloured Lie algebras and their $q$-deformations have attracted a fair amount of interest over the years, as witnessed for instance by the papers $[5,6,8,11,19,23,24,26]$. In [5, 11, 24], *-representations by bounded and unbounded operators on a Hilbert space are described for the coloured analogues of the Lie algebra $\mathfrak{s l}(2 ; \mathbb{C})$ and of the Lie algebra of the group of plane motions, two other important examples of non-commutative, three-dimensional coloured Lie algebras. The latter case is generalized to $q$-deformations in [26].

A different approach is taken in [23], where operator representations of the coloured Heisenberg-Lie commutation relations are constructed using power series of representations of Heisenberg's canonical commutation relation (1.2). By choosing various specific pairs of operators $a$ and $b$ satisfying (1.2) and substituting them into the power series for $b_{2}$ and $b_{3}$, classes of specific operator representations are found. They are then shown to lead to non-trivial functional differential-difference interpolation and combinatorial identities involving Euler, Bernoulli, and Stirling numbers. Most of the operators arising in these representations are unbounded; this is perhaps not surprising in the light of the WintnerWielandt theorem.

For $q_{1}=q_{2}=-1$, it is immediate from (1.1) that $b_{3}^{2}$ commutes with $b_{1}$ and $b_{2}$, so that $b_{3}^{2}$ is a central element of the unital algebra generated by $b_{1}, b_{2}$, and $b_{3}$. Assuming that the centre is trivial (a condition that arises naturally for instance in the context of irreducibility and Schur's Lemma), this implies that $b_{3}^{2}$ is a scalar multiple of the identity. As observed in [23], this cannot happen in a unital normed algebra unless $b_{3}^{2}=0$. Indeed, assume towards a contradiction that $\mathscr{A}$ is a unital normed algebra containing elements $b_{1}, b_{2}$, and $b_{3}$ which satisfy (1.1) with $q_{1}=q_{2}=-1$ and such that $b_{3}^{2}=\alpha 1_{\mathscr{A}}$ for some $\alpha \in \mathbb{C} \backslash\{0\}$. The first relation in (1.1) implies that $b_{1} b_{2} b_{3}+b_{2} b_{1} b_{3}=b_{3}^{2}=\alpha 1_{\mathscr{A}}$. Since $b_{1} b_{3}=-b_{3} b_{1}$ by the second relation, we have $b_{1}\left(b_{2} b_{3}\right)-\left(b_{2} b_{3}\right) b_{1}=\alpha 1_{\mathscr{A}}$, and so the elements $a=b_{1}$ and $b=\alpha^{-1} b_{2} b_{3}$ satisfy Heisenberg's canonical commutation relation (1.2), contradicting the Wintner-Wielandt theorem. Note that Example 1.1 is in accordance with this observation, since in this example we have $b_{3}^{2}=0$, so that $b_{3}^{2}$ does not satisfy the initial assumption of being a non-zero scalar multiple of the identity.

As the results described above indicate, a better understanding of how one can realize the $\left(q_{1}, q_{2}\right)$-deformed Heisenberg-Lie commutation relations inside a normed algebra is needed. We resolve this problem in the present paper by constructing a Banach algebra which contains a universal normalized solution to the $\left(q_{1}, q_{2}\right)$-deformed Heisenberg-Lie commutation relations in the following specific sense.

1.2 Theorem. For each pair $q_{1}, q_{2} \in \mathbb{C} \backslash\{0\}$, there is a unital Banach algebra $\mathscr{B}_{q_{1}, q_{2}}$ containing elements $b_{1}, b_{2}$, and $b_{3}$ satisfying the $\left(q_{1}, q_{2}\right)$-deformed Heisenberg-Lie commutation relations and such that

(i) $\left\|b_{1}\right\|=\left\|b_{2}\right\|=1$ and $\left\|b_{3}\right\|=1+\left|q_{1}\right|$; 
(ii) whenever $\mathscr{A}$ is a unital Banach algebra containing elements $a_{1}, a_{2}$, and $a_{3}$ satisfying the $\left(q_{1}, q_{2}\right)$-deformed Heisenberg-Lie commutation relations and such that $\left\|a_{1}\right\| \leqslant 1$ and $\left\|a_{2}\right\| \leqslant 1$, there is a unique bounded unital algebra homomorphism $\varphi: \mathscr{B}_{q_{1}, q_{2}} \rightarrow \mathscr{A}$ with $\varphi\left(b_{j}\right)=a_{j}$ for $j=1,2,3$; further, $\|\varphi\|=1$.

When interpreting (i), note that $1+\left|q_{1}\right|$ is the largest possible value of the norm of $b_{3}$, given that $\left\|b_{1}\right\|=\left\|b_{2}\right\|=1$ and $b_{3}=b_{1} b_{2}-q_{1} b_{2} b_{1}$.

We shall also consider the natural counterpart of the $q$-deformed Heisenberg-Lie commutation relations for $*$-algebras. Before making this precise, we recall some standard definitions.

1.3 Definition. A map $a \mapsto a^{*}$ on a complex algebra $\mathscr{A}$ is an involution if it is conjugate linear, antimultiplicative, and has period two, that is,

$$
(\alpha a+b)^{*}=\bar{\alpha} a^{*}+b^{*}, \quad(a b)^{*}=b^{*} a^{*}, \quad \text { and } \quad\left(a^{*}\right)^{*}=a \quad(\alpha \in \mathbb{C}, a, b \in \mathscr{A}) .
$$

A complex algebra with an involution is a *-algebra.

Now suppose that $\mathscr{A}$ is a Banach algebra with an involution. We say that $\mathscr{A}$ is a Banach $*$-algebra if the involution is isometric, that is, if $\left\|a^{*}\right\|=\|a\|$ for each $a \in \mathscr{A}$; and we say that $\mathscr{A}$ is a $C^{*}$-algebra if $\left\|a^{*} a\right\|=\|a\|^{2}$ for each $a \in \mathscr{A}$.

For non-zero real parameters $q_{1}$ and $q_{2}$ and elements $c_{1}$ and $c_{2}$ of a $*$-algebra, the relations

$$
c_{1} c_{1}^{*}-q_{1} c_{1}^{*} c_{1}=c_{2} \quad \text { and } \quad q_{2} c_{1} c_{2}-c_{2} c_{1}=0
$$

are the natural $*$-analogue of (1.1). The correspondence is given by $b_{1}=c_{1}, b_{2}=c_{1}^{*}$, and $b_{3}=c_{2}$. (To be precise, (1.3) states that these three elements satisfy the first two relations in (1.1), while taking adjoints in (1.3) and using that $q_{1}$ and $q_{2}$ are real shows that $c_{2}$ is self-adjoint and that the third relation in (1.1) holds). We call (1.3) the $*$-algebraic $\left(q_{1}, q_{2}\right)$-deformed Heisenberg-Lie commutation relations, and we obtain the following counterpart of Theorem 1.2.

1.4 Theorem. For each pair $q_{1}, q_{2} \in \mathbb{R} \backslash\{0\}$, there is a unital Banach $*$-algebra $\mathscr{C}_{q_{1}, q_{2}}$ containing elements $c_{1}$ and $c_{2}$ satisfying the $*$-algebraic $\left(q_{1}, q_{2}\right)$-deformed Heisenberg-Lie commutation relations and such that

(i) $\left\|c_{1}\right\|=1$ and $\left\|c_{2}\right\|=1+\left|q_{1}\right|$;

(ii) whenever $\mathscr{A}$ is a unital Banach *-algebra containing elements $a_{1}$ and $a_{2}$ satisfying the $*$-algebraic $\left(q_{1}, q_{2}\right)$-deformed Heisenberg-Lie commutation relations and such that $\left\|a_{1}\right\| \leqslant 1$, there is a unique bounded unital $*$-homomorphism $\varphi: \mathscr{C}_{q_{1}, q_{2}} \rightarrow \mathscr{A}$ with $\varphi\left(c_{j}\right)=a_{j}$ for $j=1,2$; further, $\|\varphi\|=1$.

Theorems 1.2 and 1.4 are proved in Section 2. The following theorem, which summarizes the results of Section 3, explains why Theorem 1.4 is concerned with Banach $*$-algebras, not $C^{*}$-algebras (which would be the conventional choice when studying commutation relations, going back to the quantum-mechanical origins of the subject). 
1.5 Theorem. Let $c_{1}$ and $c_{2}$ be elements of a $C^{*}$-algebra.

(i) Suppose that one of the following five conditions holds:

- $q_{1}, q_{2} \in(-\infty, 0)$;

- $q_{1} \in(0,1)$ and $q_{2} \in\left(-\left(1-q_{1}\right) /\left(1+q_{1}\right), 0\right) \cup(0,1)$;

- $q_{1} \in(1, \infty)$ and $q_{2} \in\left(-\infty,-\left(q_{1}+1\right) /\left(q_{1}-1\right)\right) \cup(1, \infty)$;

- $q_{2} \in(0,1)$ and $q_{1} \in\left(-\left(1-q_{2}\right) /\left(1+q_{2}\right), 0\right)$;

- $q_{2} \in(1, \infty)$ and $q_{1} \in\left(-\infty,-\left(q_{2}+1\right) /\left(q_{2}-1\right)\right)$.

Then the elements $c_{1}$ and $c_{2}$ satisfy the $*$-algebraic $\left(q_{1}, q_{2}\right)$-deformed Heisenberg-Lie commutation relations if and only if $c_{1}=c_{2}=0$.

(ii) The elements $c_{1}$ and $c_{2}$ satisfy the *-algebraic $(1,1)$-deformed Heisenberg-Lie commutation relations if and only if $c_{1}$ is normal and $c_{2}=0$.

This result raises the question of whether or not a pair of operators on a Hilbert space can satisfy the $*$-algebraic $\left(q_{1}, q_{2}\right)$-deformed Heisenberg-Lie commutation relations in a 'non-trivial' way for parameters $q_{1}, q_{2} \in \mathbb{R} \backslash\{0\}$ other than those mentioned in Theorem 1.5. We address this question in Section 4 , where we determine all pairs $q_{1}, q_{2} \in \mathbb{R} \backslash\{0\}$ and weights $\omega \in \ell_{\infty}(\mathbb{Z})$ such that the corresponding weighted right-shift operator $R_{\omega}$ on $\ell_{2}(\mathbb{Z})$ together with the operator $R_{\omega} R_{\omega}^{*}-q_{1} R_{\omega}^{*} R_{\omega}$ satisfy the $*$-algebraic $\left(q_{1}, q_{2}\right)$-deformed Heisenberg-Lie commutation relations.

\section{The proofs of Theorems 1.2 and 1.4}

Our first aim is to construct a Banach algebra $\mathscr{B}_{q_{1}, q_{2}}$ with the properties listed in Theorem 1.2. As it turns out, we shall define $\mathscr{B}_{q_{1}, q_{2}}$ as a quotient of a certain semigroup Banach algebra, so we begin with some general facts about such algebras.

2.1 Semigroup Banach algebras. Let $S$ be a semigroup. The Banach space

$$
\ell_{1}(S)=\left\{f: S \rightarrow \mathbb{C}\left|\|f\|_{1} \stackrel{\text { def }}{=} \sum_{s \in S}\right| f(s) \mid<\infty\right\},
$$

equipped with pointwise defined vector-space operations and norm $\|\cdot\|_{1}$, is a Banach algebra for the convolution product $\star$ defined as follows. For each $s \in S$, let $\delta_{s}$ be the point mass at $s$. Then each element $f$ of $\ell_{1}(S)$ can be expressed uniquely as an absolutely convergent sum $f=\sum_{s \in S} f(s) \delta_{s}$, and the convolution product is determined by the formula $\delta_{s} \star \delta_{t}=\delta_{s t}$ for each $s, t \in S$. We call $\ell_{1}(S)$ the semigroup Banach algebra of $S$. In the case where the semigroup $S$ has a neutral element $e$, the element $\delta_{e}$ is an identity in the algebra $\ell_{1}(S)$.

2.2 The free semigroup on two generators. Let $\mathbb{S}_{2}$ be the free unital semigroup on two generators $s_{1}$ and $s_{2}$. We write $e$ for the neutral element of $\mathbb{S}_{2}$. There is a natural notion of length of an element of $\mathbb{S}_{2}$, namely

$$
\operatorname{len}(e)=0 \quad \text { and } \quad \operatorname{len}\left(s_{j_{1}} s_{j_{2}} \cdots s_{j_{n}}\right)=n \quad\left(n \in \mathbb{N}, j_{1}, j_{2}, \ldots, j_{n} \in\{1,2\}\right) .
$$


For each $n \in \mathbb{N}_{0}$ (the set of non-negative integers), let $\mathbb{W}_{n}=\left\{w \in \mathbb{S}_{2} \mid \operatorname{len}(w)=n\right\}$ and define

$$
P_{n}: f \mapsto \sum_{w \in \mathbb{W}_{n}} f(w) \delta_{w}, \quad \ell_{1}\left(\mathbb{S}_{2}\right) \rightarrow \ell_{1}\left(\mathbb{S}_{2}\right)
$$

Then $P_{n}$ is an idempotent operator of norm 1 . Since $\left\{\mathbb{W}_{n}\right\}_{n=0}^{\infty}$ is a partition of $\mathbb{S}_{2}$, we have

$$
\|f\|_{1}=\sum_{n=0}^{\infty}\left\|P_{n} f\right\|_{1} \quad \text { and } \quad f=\sum_{n=0}^{\infty} P_{n} f \quad \text { (absolute convergence) } \quad\left(f \in \ell_{1}\left(\mathbb{S}_{2}\right)\right)
$$

and moreover

$$
P_{m} P_{n}=\left\{\begin{array}{ll}
P_{n} & \text { if } m=n \\
0 & \text { otherwise }
\end{array} \quad \text { and } \quad\left(\operatorname{im} P_{m}\right) \star\left(\operatorname{im} P_{n}\right) \subseteq \operatorname{im} P_{m+n} \quad\left(m, n \in \mathbb{N}_{0}\right),\right.
$$

where im $P_{m}$ denotes the image of the operator $P_{m}$. The identities (2.1)-(2.2) imply that $\left(P_{n}\right)_{n=0}^{\infty}$ is a grading of $\ell_{1}\left(\mathbb{S}_{2}\right)$.

2.3 Lemma. For each $N \in \mathbb{N}_{0}$, the set

$$
\bigcap_{m=0}^{N} \operatorname{ker} P_{m}=\left\{\sum_{n=N+1}^{\infty}\left(\sum_{w \in \mathbb{W}_{n}} \alpha_{w} \delta_{w}\right) \mid \alpha_{w} \in \mathbb{C} \quad\left(w \in \mathbb{S}_{2}\right), \sum_{n=N+1}^{\infty}\left(\sum_{w \in \mathbb{W}_{n}}\left|\alpha_{w}\right|\right)<\infty\right\}
$$

is a closed two-sided ideal in $\ell_{1}\left(\mathbb{S}_{2}\right)$.

Proof. Set $\mathscr{I}=\bigcap_{m=0}^{N}$ ker $P_{m}$. This is a closed linear subspace of $\ell_{1}\left(\mathbb{S}_{2}\right)$ because ker $P_{m}$ is a closed linear subspace of $\ell_{1}\left(\mathbb{S}_{2}\right)$ for each $m \in \mathbb{N}_{0}$. To see that $\mathscr{I}$ is a left ideal in $\ell_{1}\left(\mathbb{S}_{2}\right)$, let $f \in \ell_{1}\left(\mathbb{S}_{2}\right)$ and $g \in \mathscr{I}$. Then we have

$$
f \star g=\left(\sum_{m=0}^{\infty} P_{m} f\right) \star\left(\sum_{n=N+1}^{\infty} P_{n} g\right)=\sum_{m=0}^{\infty}\left(\sum_{n=N+1}^{\infty} P_{m} f \star P_{n} g\right) ;
$$

(2.2) implies that $P_{m} f \star P_{n} g \in \operatorname{im} P_{m+n} \subseteq \operatorname{ker} P_{k}$ whenever $m \in \mathbb{N}_{0}$ and $n>N \geqslant k$, and so $f \star g \in \mathscr{I}$. A similar argument shows that $\mathscr{I}$ is a right ideal in $\ell_{1}\left(\mathbb{S}_{2}\right)$.

2.4 Lemma. Given two elements $a_{1}$ and $a_{2}$ in the unit ball of a unital Banach algebra $\mathscr{A}$, there is a unique bounded unital algebra homomorphism $\theta: \ell_{1}\left(\mathbb{S}_{2}\right) \rightarrow \mathscr{A}$ such that $\theta\left(\delta_{s_{j}}\right)=$ $a_{j}$ for $j=1,2$. Further, $\|\theta\|=1$.

Proof. We begin by defining $\theta\left(\delta_{e}\right)=1_{\mathscr{A}}$ (the identity of $\mathscr{A}$ ) and

$$
\theta\left(\delta_{s_{j_{1}} s_{j_{2}} \cdots s_{j_{n}}}\right)=a_{j_{1}} a_{j_{2}} \cdots a_{j_{n}} \quad\left(n \in \mathbb{N}, j_{1}, j_{2}, \ldots, j_{n} \in\{1,2\}\right) .
$$

Since $a_{1}$ and $a_{2}$ both have norm at most 1 , this is also the case for $\theta\left(\delta_{w}\right)$ for each $w \in \mathbb{S}_{2}$. Hence we can extend $\theta$ to all of $\ell_{1}\left(\mathbb{S}_{2}\right)$ by linearity and continuity, and this definition makes $\theta$ multiplicative and contractive; in fact $\|\theta\|=1$ because $\theta$ is unital. 
To prove the uniqueness statement, let $\varphi: \ell_{1}\left(\mathbb{S}_{2}\right) \rightarrow \mathscr{A}$ be any bounded unital algebra homomorphism with $\varphi\left(\delta_{s_{j}}\right)=a_{j}$ for $j=1,2$. Then $\varphi\left(\delta_{e}\right)=1_{\mathscr{A}}=\theta\left(\delta_{e}\right)$ and

$$
\varphi\left(\delta_{s_{j_{1}} s_{j_{2}} \cdots s_{j_{n}}}\right)=\varphi\left(\delta_{s_{j_{1}}} \star \delta_{s_{j_{2}}} \star \cdots \star \delta_{s_{j_{n}}}\right)=a_{j_{1}} a_{j_{2}} \cdots a_{j_{n}}=\theta\left(\delta_{s_{j_{1}} s_{j_{2}} \cdots s_{j_{n}}}\right)
$$

for each $n \in \mathbb{N}$ and $j_{1}, j_{2}, \ldots, j_{n} \in\{1,2\}$, and so $\varphi=\theta$ by linearity and continuity.

Proof of Theorem 1.2. Consider the following five elements of $\ell_{1}\left(\mathbb{S}_{2}\right)$ :

$$
\begin{array}{lll}
f_{1}=\delta_{s_{1}}, & f_{2}=\delta_{s_{2}}, & f_{3}=f_{1} \star f_{2}-q_{1} f_{2} \star f_{1}, \\
g_{1}=q_{2} f_{1} \star f_{3}-f_{3} \star f_{1}, & \text { and } & g_{2}=f_{2} \star f_{3}-q_{2} f_{3} \star f_{2} .
\end{array}
$$

Let $\mathscr{J}$ denote the closed two-sided ideal in $\ell_{1}\left(\mathbb{S}_{2}\right)$ generated by $g_{1}$ and $g_{2}$, and define $\mathscr{B}_{q_{1}, q_{2}}=\ell_{1}\left(\mathbb{S}_{2}\right) / \mathscr{J}$ and $b_{j}=\pi\left(f_{j}\right)$ for $j=1,2,3$, where $\pi: \ell_{1}\left(\mathbb{S}_{2}\right) \rightarrow \mathscr{B}_{q_{1}, q_{2}}$ is the quotient homomorphism. It follows immediately from (2.3)-(2.4) and the definition of $\mathscr{J}$ that the elements $b_{1}, b_{2}$, and $b_{3}$ satisfy (1.1).

To show that their norms are as stated in Theorem 1.2(i), we recall that the quotient norm on $\mathscr{B}_{q_{1}, q_{2}}$ is given by

$$
\|\pi(f)\|=\inf \left\{\|f-g\|_{1} \mid g \in \mathscr{J}\right\} \quad\left(f \in \ell_{1}\left(\mathbb{S}_{2}\right)\right) .
$$

In particular, we have $\left\|b_{j}\right\| \leqslant\left\|f_{j}\right\|_{1}$, so that $\left\|b_{1}\right\|,\left\|b_{2}\right\| \leqslant 1$ and

$$
\left\|b_{3}\right\| \leqslant\left\|f_{3}\right\|_{1}=\left\|\delta_{s_{1} s_{2}}-q_{1} \delta_{s_{2} s_{1}}\right\|_{1}=1+\left|q_{1}\right| .
$$

For the converse inequalities, we note that $g_{1}, g_{2} \in \operatorname{im} P_{3} \subseteq \bigcap_{n=0}^{2} \operatorname{ker} P_{n}$ by (2.2), and so $\mathscr{J} \subseteq \bigcap_{n=0}^{2} \operatorname{ker} P_{n}$ by Lemma 2.3. Since $\left\|P_{1}\right\|=\left\|P_{2}\right\|=1$, it follows that

$$
\left\|f_{j}-g\right\|_{1} \geqslant\left\|P_{1}\left(f_{j}-g\right)\right\|_{1}=\left\|f_{j}\right\|_{1}=1 \quad(j=1,2)
$$

and

$$
\left\|f_{3}-g\right\|_{1} \geqslant\left\|P_{2}\left(f_{3}-g\right)\right\|_{1}=\left\|f_{3}\right\|_{1}=1+\left|q_{1}\right|
$$

for each $g \in \mathscr{J}$. This implies that $\left\|b_{1}\right\|,\left\|b_{2}\right\| \geqslant 1$ and $\left\|b_{3}\right\| \geqslant 1+\left|q_{1}\right|$, as required.

Finally, to establish Theorem 1.2(ii), suppose that $a_{1}, a_{2}$, and $a_{3}$ are elements of a unital Banach algebra $\mathscr{A}$ satisfying $a_{1} a_{2}-q_{1} a_{2} a_{1}=a_{3}, q_{2} a_{1} a_{3}-a_{3} a_{1}=0, a_{2} a_{3}-q_{2} a_{3} a_{2}=0$, and $\left\|a_{1}\right\|,\left\|a_{2}\right\| \leqslant 1$. By Lemma 2.4, there is a unique bounded unital algebra homomorphism $\theta: \ell_{1}\left(\mathbb{S}_{2}\right) \rightarrow \mathscr{A}$ with $\theta\left(f_{j}\right)=a_{j}$ for $j=1,2$, and $\|\theta\|=1$. We have

$$
\theta\left(f_{3}\right)=\theta\left(f_{1}\right) \theta\left(f_{2}\right)-q_{1} \theta\left(f_{2}\right) \theta\left(f_{1}\right)=a_{1} a_{2}-q_{1} a_{2} a_{1}=a_{3}
$$

and thus

$$
\theta\left(g_{1}\right)=q_{2} \theta\left(f_{1}\right) \theta\left(f_{3}\right)-\theta\left(f_{3}\right) \theta\left(f_{1}\right)=q_{2} a_{1} a_{3}-a_{3} a_{1}=0 ;
$$

similarly, we see that $\theta\left(g_{2}\right)=0$. Hence $\mathscr{J} \subseteq \operatorname{ker} \theta$, so the first isomorphism theorem implies that there is a unique bounded unital algebra homomorphism $\varphi: \mathscr{B}_{q_{1}, q_{2}} \rightarrow \mathscr{A}$ such that 
$\varphi \circ \pi=\theta$, and $\|\varphi\|=\|\theta\|=1$. In particular, it follows that $\varphi\left(b_{j}\right)=\varphi\left(\pi\left(f_{j}\right)\right)=\theta\left(f_{j}\right)=a_{j}$ for $j=1,2,3$, as required.

To prove the uniqueness statement, observe that if $\psi: \mathscr{B}_{q_{1}, q_{2}} \rightarrow \mathscr{A}$ is a bounded unital algebra homomorphism with $\psi\left(b_{j}\right)=a_{j}$ for $j=1,2$, then $\psi \circ \pi: \ell_{1}\left(\mathbb{S}_{2}\right) \rightarrow \mathscr{A}$ is a bounded unital algebra homomorphism with $\psi \circ \pi\left(f_{j}\right)=\psi\left(b_{j}\right)=a_{j}$ for $j=1,2$. Hence $\psi \circ \pi=\theta$ by the uniqueness of $\theta$, and so $\psi=\varphi$ by the uniqueness of $\varphi$.

2.5 Remark. In the case where $q_{1}=1$, the Wintner-Wielandt theorem implies that the element $b_{3}$ constructed in the proof above cannot be a non-zero scalar multiple of the identity (because $b_{3}=b_{1} b_{2}-b_{2} b_{1}$ ). In fact this is true no matter what values $q_{1}, q_{2} \in \mathbb{C} \backslash\{0\}$ take, since for each $\alpha \in \mathbb{C}$ we have

$$
\left\|b_{3}-\alpha 1_{\mathscr{B}}\right\|=\left\|f_{3}-\alpha \delta_{e}\right\|_{1}=1+\left|q_{1}\right|+|\alpha|>1,
$$

where $1_{\mathscr{B}}=\pi\left(\delta_{e}\right)$ denotes the identity of $\mathscr{B}_{q_{1}, q_{2}}$. Only the first equality in (2.5) is not obvious; we prove it by a slight refinement of the argument used in the calculation of the norm of $b_{3}$ above. The fact that $b_{3}-\alpha 1_{\mathscr{B}}=\pi\left(f_{3}-\alpha \delta_{e}\right)$ implies that $\left\|b_{3}-\alpha 1_{\mathscr{B}}\right\| \leqslant$ $\left\|f_{3}-\alpha \delta_{e}\right\|_{1}$. Conversely, since $P_{0}+P_{2}$ is an operator of norm 1 , we have

$$
\left\|f_{3}-\alpha \delta_{e}-g\right\|_{1} \geqslant\left\|\left(P_{0}+P_{2}\right)\left(f_{3}-\alpha \delta_{e}-g\right)\right\|_{1}=\left\|f_{3}-\alpha \delta_{e}\right\|_{1} \quad(g \in \mathscr{J}),
$$

and therefore $\left\|b_{3}-\alpha 1_{\mathscr{B}}\right\| \geqslant\left\|f_{3}-\alpha \delta_{e}\right\|_{1}$; this completes the proof of (2.5).

2.6 The involution on $\boldsymbol{\ell}_{\mathbf{1}}\left(\mathbf{S}_{\mathbf{2}}\right)$. As explained in [4, example 3.1.4(iv)], the definitions $e^{*}=e, s_{1}^{*}=s_{2}, s_{2}^{*}=s_{1}$, and

$$
\left(s_{j_{1}} s_{j_{2}} \cdots s_{j_{n-1}} s_{j_{n}}\right)^{*}=s_{j_{n}}^{*} s_{j_{n-1}}^{*} \cdots s_{j_{2}}^{*} s_{j_{1}}^{*} \quad\left(n \geqslant 2, j_{1}, j_{2}, \ldots, j_{n-1}, j_{n} \in\{1,2\}\right)
$$

give an antimultiplicative mapping of period two on $\mathbb{S}_{2}$. This induces an isometric involution on $\ell_{1}\left(\mathbb{S}_{2}\right)$ by the rule $\delta_{w}^{*}=\delta_{w^{*}}$ for each $w \in \mathbb{S}_{2}$, and so $\ell_{1}\left(\mathbb{S}_{2}\right)$ is a Banach $*$-algebra.

With respect to this involution, the following *-analogue of Lemma 2.4 holds.

2.7 Lemma. Given an element $a$ in the unit ball of a unital Banach $*$-algebra $\mathscr{A}$, there is a unique bounded unital $*$-homomorphism $\theta: \ell_{1}\left(\mathbb{S}_{2}\right) \rightarrow \mathscr{A}$ such that $\theta\left(\delta_{s_{1}}\right)=a$. Further, $\|\theta\|=1$.

Proof. Taking $a_{1}=a$ and $a_{2}=a^{*}$ in Lemma 2.4, we see that there is a unique bounded unital algebra homomorphism $\theta: \ell_{1}\left(\mathbb{S}_{2}\right) \rightarrow \mathscr{A}$ with $\theta\left(\delta_{s_{j}}\right)=a_{j}$ for $j=1,2$, and $\|\theta\|=1$. A straightforward calculation shows that $\theta$ is a $*$-homomorphism.

To prove the uniqueness statement, suppose that $\varphi: \ell_{1}\left(\mathbb{S}_{2}\right) \rightarrow \mathscr{A}$ is any bounded unital $*$-homomorphism with $\varphi\left(\delta_{s_{1}}\right)=a\left(=a_{1}\right)$. Then $\varphi\left(\delta_{s_{2}}\right)=\varphi\left(\delta_{s_{1}}^{*}\right)=\varphi\left(\delta_{s_{1}}\right)^{*}=a^{*}=a_{2}$, and so $\varphi=\theta$ by the uniqueness of $\theta$ (as stated in Lemma 2.4).

2.8 Lemma. Let $q_{1}, q_{2} \in \mathbb{R} \backslash\{0\}$. Then the Banach algebra $\mathscr{B}_{q_{1}, q_{2}}$ constructed in the proof of Theorem 1.2 has an isometric involution such that $b_{1}^{*}=b_{2}$. 
Proof. The adjoints of the elements of $\ell_{1}\left(\mathbb{S}_{2}\right)$ defined in $(2.3)-(2.4)$ are given by $f_{1}^{*}=f_{2}$, $f_{3}^{*}=f_{3}$, and $g_{1}^{*}=-g_{2}$. Hence the closed two-sided ideal $\mathscr{J}$ generated by $g_{1}$ and $g_{2}$ is automatically a $*$-ideal, and so we can define an involution on $\mathscr{B}_{q_{1}, q_{2}}$ by $\pi(f)^{*}=\pi\left(f^{*}\right)$ for each $f \in \ell_{1}\left(\mathbb{S}_{2}\right)$. In particular, we have $b_{1}^{*}=\pi\left(f_{1}\right)^{*}=\pi\left(f_{1}^{*}\right)=\pi\left(f_{2}\right)=b_{2}$ and

$$
\begin{aligned}
\left\|\pi(f)^{*}\right\| & =\left\|\pi\left(f^{*}\right)\right\|=\inf \left\{\left\|f^{*}-g\right\|_{1} \mid g \in \mathscr{J}\right\} \\
& =\inf \left\{\left\|f^{*}-g^{*}\right\|_{1} \mid g \in \mathscr{J}\right\}=\inf \left\{\|f-g\|_{1} \mid g \in \mathscr{J}\right\}=\|\pi(f)\|
\end{aligned}
$$

for each $f \in \ell_{1}\left(\mathbb{S}_{2}\right)$, as required.

Proof of Theorem 1.4. Theorem 1.2 and Lemma 2.8 imply that the elements $c_{1}=b_{1}$ and $c_{2}=b_{3}$ of the unital Banach $*$-algebra $\mathscr{C}_{q_{1}, q_{2}}=\mathscr{B}_{q_{1}, q_{2}}$ satisfy (1.3) and Theorem 1.4(i). The proof of Theorem 1.4(ii) is similar to that of Theorem 1.2(ii), just with the reference to Lemma 2.4 replaced by a reference to Lemma 2.7 .

\section{The $*$-algebraic Heisenberg-Lie commutation rela- tions in $C^{*}$-algebras}

The main aim of this section is to show that, for many parameters $q_{1}, q_{2} \in \mathbb{R} \backslash\{0\}$, the *-algebraic $\left(q_{1}, q_{2}\right)$-deformed Heisenberg-Lie commutation relations can only be realized 'trivially' in a $C^{*}$-algebra (where the exact meaning of 'trivially' will depend on the context). Several of our results apply to more general types of $*$-algebras.

We begin with the classical case, where $q_{1}=q_{2}=1$.

For a complex algebra $\mathscr{A}$, we define its conditional unitization $\mathscr{A}^{\sharp}$ to be $\mathscr{A}$ with an

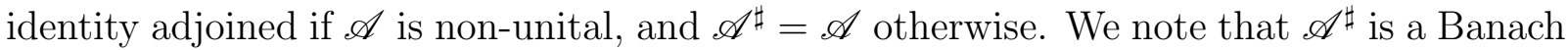
algebra whenever $\mathscr{A}$ is a Banach algebra and that an involution on $\mathscr{A}$ extends uniquely to $\mathscr{A}^{\sharp}$.

An element $a$ of a Banach algebra $\mathscr{A}$ is quasi-nilpotent if its spectrum consists of 0 only, that is, if $a-\alpha 1_{\mathscr{A} \sharp}$ is invertible in $\mathscr{A}^{\sharp}$ for each $\alpha \in \mathbb{C} \backslash\{0\}$ (where $1_{\mathscr{A}}$ denotes the identity of $\mathscr{A}^{\sharp}$ ). The Kleinecke-Shirokov theorem (as stated in [7, problem 232] or [4, theorem 2.7.19]) implies that if $b_{1}, b_{2}$, and $b_{3}$ are elements of a Banach algebra satisfying the classical Heisenberg-Lie commutation relations, then $b_{3}$ is necessarily quasi-nilpotent. This has an important consequence in the $*$-algebraic case as we shall show next, using the following standard concepts.

3.1 Definition. Let $\mathscr{A}$ be a $*$-algebra. A linear functional $\lambda: \mathscr{A} \rightarrow \mathbb{C}$ is positive if $\left\langle a^{*} a, \lambda\right\rangle \geqslant 0$ for each $a \in \mathscr{A}$. The $*$-radical of $\mathscr{A}$ is given by

$$
*-\operatorname{rad} \mathscr{A}=\bigcap\left\{\operatorname{ker} \lambda \mid \lambda: \mathscr{A}^{\sharp} \rightarrow \mathbb{C} \text { is positive }\right\} .
$$

If $*-\operatorname{rad} \mathscr{A}=\{0\}$, then $\mathscr{A}$ is $*$-semisimple. 
The $*$-radical is a $*$-ideal. For a Banach $*$-algebra $\mathscr{A}$, its $*$-radical can be viewed as the obstruction to representing $\mathscr{A}$ faithfully on a Hilbert space in the following precise sense: $\mathscr{A}$ is $*$-semisimple if and only if there exist a Hilbert space $H$ and an injective $*$-homomorphism from $\mathscr{A}$ into $\mathscr{B}(H)$ (the $C^{*}$-algebra of all bounded linear operators on $H$ ); see $[4$, theorem 3.1.17] for details. In particular, every $C^{*}$-algebra is $*$-semisimple (e.g., see [4, corollary 3.2.13]).

The *-radical and quasi-nilpotent elements are related through the following result which is an immediate consequence of [4, corollary 3.1.6(ii)].

3.2 Lemma. Let $a$ be an element of a Banach algebra $\mathscr{A}$ with an involution. If $a^{*} a$ is quasi-nilpotent, then $a \in *-\operatorname{rad} \mathscr{A}$.

3.3 Proposition. Let $c_{1}$ and $c_{2}$ be elements of a Banach algebra $\mathscr{A}$ with an involution. If $c_{1}$ and $c_{2}$ satisfy the $*$-algebraic $(1,1)$-deformed Heisenberg-Lie commutation relations, then $c_{2} \in *-\operatorname{rad} \mathscr{A}$.

Proof. By assumption, $c_{1}, c_{1}^{*}$, and $c_{2}$ satisfy the classical Heisenberg-Lie commutation relations, so as already mentioned, the Kleinecke-Shirokov theorem implies that $c_{2}$ is quasinilpotent; consequently $c_{2}^{2}$ is also quasi-nilpotent. Now the result follows from Lemma 3.2 because $c_{2}$ is self-adjoint, so that $c_{2}^{2}=c_{2}^{*} c_{2}$.

An element $a$ of a $*$-algebra is normal if it commutes with its adjoint, that is, if $a a^{*}=a^{*} a$. We can now describe all solutions to the $*$-algebraic $(1,1)$-deformed Heisenberg-Lie commutation relations in the $*$-semisimple case as follows.

3.4 Corollary. Let $c_{1}$ and $c_{2}$ be elements of a $*$-semisimple Banach algebra $\mathscr{A}$ with an involution. Then $c_{1}$ and $c_{2}$ satisfy the $*$-algebraic $(1,1)$-deformed Heisenberg-Lie commutation relations if and only if $c_{1}$ is normal and $c_{2}=0$.

Proof. ' $\Rightarrow$ '. Proposition 3.3 together with the $*$-semisimplicity of $\mathscr{A}$ imply that $c_{2}=0$. Since $c_{2}=c_{1} c_{1}^{*}-q_{1} c_{1}^{*} c_{1}$ and $q_{1}=1$, we conclude that $c_{1}$ is normal.

The converse is immediate.

Having thus settled the classical case, we proceed to consider other parameters, starting with the following simple observation.

3.5 Lemma. Let $q_{1}, q_{2} \in \mathbb{R} \backslash\{0\}$, and let $c$ be an element of a $*$-algebra. The following five conditions are equivalent:

(a) $q_{1} c^{*} c^{2}+q_{2} c^{2} c^{*}=\left(1+q_{1} q_{2}\right) c c^{*} c$;

(b) $c_{1}=c$ and $c_{2}=c c^{*}-q_{1} c^{*} c$ satisfy the $*$-algebraic $\left(q_{1}, q_{2}\right)$-deformed Heisenberg-Lie commutation relations;

(c) $c_{1}=c$ and $c_{2}=c c^{*}-q_{2}^{-1} c^{*} c$ satisfy the $*$-algebraic $\left(q_{2}^{-1}, q_{1}^{-1}\right)$-deformed HeisenbergLie commutation relations. 
(d) $c_{1}=c^{*}$ and $c_{2}=c^{*} c-q_{1}^{-1} c c^{*}$ satisfy the $*$-algebraic $\left(q_{1}^{-1}, q_{2}^{-1}\right)$-deformed HeisenbergLie commutation relations;

(e) $c_{1}=c^{*}$ and $c_{2}=c^{*} c-q_{2} c c^{*}$ satisfy the $*$-algebraic $\left(q_{2}, q_{1}\right)$-deformed Heisenberg-Lie commutation relations.

Proof. The elements $c_{1}=c$ and $c_{2}=c c^{*}-q_{1} c^{*} c$ satisfy the first relation in (1.3) by definition, while substituting them into the second relation and rearranging shows that the identities $q_{2} c_{1} c_{2}-c_{2} c_{1}=0$ and $q_{1} c^{*} c^{2}+q_{2} c^{2} c^{*}=\left(1+q_{1} q_{2}\right) c c^{*} c$ are equivalent. This proves that conditions (a) and (b) are equivalent.

The other implications can be verified in a similar fashion.

3.6 Proposition. Let $c_{1}$ and $c_{2}$ be elements of a $*$-semisimple Banach $*$-algebra $\mathscr{A}$, and let $q_{1}$ and $q_{2}$ be non-zero real numbers satisfying one of the following six conditions:

(i) $0<q_{1}<1$ and $0<q_{2}<1$;

(ii) $q_{1}>1$ and $q_{2}>1$;

(iii) $0<q_{1}<1$ and $-\frac{1-q_{1}}{1+q_{1}}<q_{2}<0$;

(iv) $0<q_{2}<1$ and $-\frac{1-q_{2}}{1+q_{2}}<q_{1}<0$;

(v) $q_{1}>1$ and $q_{2}<-\frac{q_{1}+1}{q_{1}-1}(<-1)$;

(vi) $q_{2}>1$ and $q_{1}<-\frac{q_{2}+1}{q_{2}-1}(<-1)$.

Then $c_{1}$ and $c_{2}$ satisfy the $*$-algebraic $\left(q_{1}, q_{2}\right)$-deformed Heisenberg-Lie commutation relations if and only if $c_{1}=c_{2}=0$.

Proof. Only the implication $\Rightarrow$ requires proof. By assumption, we can take a Hilbert space $H$ and an injective $*$-homomorphism $\varphi: \mathscr{A} \rightarrow \mathscr{B}(H)$. Since $c_{1}$ satisfies the identity in Lemma 3.5(a), the same is true for $c=\varphi\left(c_{1}\right) \in \mathscr{B}(H)$. Right-multiplying this identity by $c^{*}$ and applying standard properties of the norm on $\mathscr{B}(H)$ yields

$$
\left(\left|q_{1}\right|+\left|q_{2}\right|\right)\|c\|^{4} \geqslant\left\|q_{1} c^{*} c^{2} c^{*}+q_{2} c^{2}\left(c^{*}\right)^{2}\right\|=\left\|\left(1+q_{1} q_{2}\right) c c^{*} c c^{*}\right\|=\left|1+q_{1} q_{2}\right|\|c\|^{4} .
$$

As $\varphi$ is injective, it suffices to show that $c=0$. We establish this by considering each of the six cases separately.

(i). In this case (3.1) states that $\left(q_{1}+q_{2}\right)\|c\|^{4} \geqslant\left(1+q_{1} q_{2}\right)\|c\|^{4}$, which can be rewritten as $0 \geqslant\left(1-q_{1}\right)\left(1-q_{2}\right)\|c\|^{4}$. Since $1-q_{1}$ and $1-q_{2}$ are both positive by assumption, we conclude that $c=0$, as required.

(ii). We reduce this to case (i) as follows. Lemma 3.5 implies that $c^{*}$ and $c^{*} c-q_{1}^{-1} c c^{*}$ satisfy the $*$-algebraic $\left(q_{1}^{-1}, q_{2}^{-1}\right)$-deformed Heisenberg-Lie commutation relations. Since $q_{1}^{-1}, q_{2}^{-1} \in(0,1)$, case (i) implies that $c^{*}=0$, and thus $c=0$. 
(iii). We have

$$
1+q_{1} q_{2}>1-q_{1} \frac{1-q_{1}}{1+q_{1}}=\frac{1+q_{1}^{2}}{1+q_{1}}>0
$$

so that (3.1) states that $\left(q_{1}-q_{2}\right)\|c\|^{4} \geqslant\left(1+q_{1} q_{2}\right)\|c\|^{4}$. Assuming that $c \neq 0$, we can cancel $\|c\|^{4}$ and rearrange to obtain

$$
0 \geqslant 1+q_{1} q_{2}+q_{2}-q_{1}=1+\left(1+q_{1}\right) q_{2}-q_{1}>1-\left(1-q_{1}\right)-q_{1}=0
$$

which is clearly absurd. Hence we conclude that $c=0$.

Finally, cases (iv), (v), and (vi) follow from Lemma 3.5 in conjunction with (iii).

To deal with the case where $q_{1}$ and $q_{2}$ are both negative, we require the following notion.

3.7 Definition. Let $\mathscr{A}$ be a $*$-algebra. We say that $\mathscr{A}$ is proper if, given $a \in \mathscr{A}$ such that $a^{*} a=0$, we have $a=0$; and we say that $\mathscr{A}$ is very proper if, given $n \in \mathbb{N}$ and $a_{1}, a_{2}, \ldots, a_{n} \in \mathscr{A}$ such that $\sum_{k=1}^{n} a_{k}^{*} a_{k}=0$, we have $a_{1}=a_{2}=\cdots=a_{n}=0$.

Note that a $*$-semisimple $*$-algebra is very proper, and a very proper $*$-algebra is proper.

3.8 Proposition. Let $c_{1}$ and $c_{2}$ be elements of a very proper *-algebra $\mathscr{A}$, and let $q_{1}, q_{2} \in(-\infty, 0)$. Then $c_{1}$ and $c_{2}$ satisfy the $*$-algebraic $\left(q_{1}, q_{2}\right)$-deformed Heisenberg-Lie commutation relations if and only if $c_{1}=c_{2}=0$.

Proof. Only the implication $\Rightarrow$ requires proof. By assumption, $c_{1}$ satisfies the identity in Lemma 3.5(a). Left-multiplying this identity by $-q_{1} q_{2}^{-1} c_{1}^{*}$ and rearranging, we obtain

$$
q_{1}^{2} c_{1}^{*} c_{1} c_{1}^{*} c_{1}-q_{1} c_{1}^{*} c_{1}^{2} c_{1}^{*}=\frac{q_{1}^{2}}{q_{2}}\left(c_{1}^{*}\right)^{2} c_{1}^{2}-\frac{q_{1}}{q_{2}} c_{1}^{*} c_{1} c_{1}^{*} c_{1}
$$

On the other hand, if we take adjoints in the identity in Lemma 3.5(a), left-multiply by $-c_{1}$, and rearrange, then we get

$$
c_{1} c_{1}^{*} c_{1} c_{1}^{*}-q_{1} c_{1}\left(c_{1}^{*}\right)^{2} c_{1}=q_{2} c_{1}^{2}\left(c_{1}^{*}\right)^{2}-q_{1} q_{2} c_{1} c_{1}^{*} c_{1} c_{1}^{*} .
$$

Now expanding the product $c_{2}^{*} c_{2}$ and substituting the above identities into the resulting expression yields

$$
\begin{aligned}
c_{2}^{*} c_{2}=\left(c_{1} c_{1}^{*}-q_{1} c_{1}^{*} c_{1}\right)^{2} & =c_{1} c_{1}^{*} c_{1} c_{1}^{*}-q_{1} c_{1}\left(c_{1}^{*}\right)^{2} c_{1}+q_{1}^{2} c_{1}^{*} c_{1} c_{1}^{*} c_{1}-q_{1} c_{1}^{*} c_{1}^{2} c_{1}^{*} \\
& =q_{2} c_{1}^{2}\left(c_{1}^{*}\right)^{2}-q_{1} q_{2} c_{1} c_{1}^{*} c_{1} c_{1}^{*}+\frac{q_{1}^{2}}{q_{2}}\left(c_{1}^{*}\right)^{2} c_{1}^{2}-\frac{q_{1}}{q_{2}} c_{1}^{*} c_{1} c_{1}^{*} c_{1},
\end{aligned}
$$

and consequently we have

$$
\begin{aligned}
0=c_{2}^{*} c_{2} & -q_{2} c_{1}^{2}\left(c_{1}^{*}\right)^{2}+q_{1} q_{2} c_{1} c_{1}^{*} c_{1} c_{1}^{*}-\frac{q_{1}^{2}}{q_{2}}\left(c_{1}^{*}\right)^{2} c_{1}^{2}+\frac{q_{1}}{q_{2}} c_{1}^{*} c_{1} c_{1}^{*} c_{1} \\
=c_{2}^{*} c_{2} & +\left(\sqrt{-q_{2}}\left(c_{1}^{*}\right)^{2}\right)^{*}\left(\sqrt{-q_{2}}\left(c_{1}^{*}\right)^{2}\right)+\left(\sqrt{q_{1} q_{2}} c_{1} c_{1}^{*}\right)^{*}\left(\sqrt{q_{1} q_{2}} c_{1} c_{1}^{*}\right) \\
& +\left(\frac{q_{1}}{\sqrt{-q_{2}}} c_{1}^{2}\right)^{*}\left(\frac{q_{1}}{\sqrt{-q_{2}}} c_{1}^{2}\right)+\left(\sqrt{\frac{q_{1}}{q_{2}}} c_{1}^{*} c_{1}\right)^{*}\left(\sqrt{\frac{q_{1}}{q_{2}}} c_{1}^{*} c_{1}\right) .
\end{aligned}
$$


As $\mathscr{A}$ is very proper, this implies that

$$
c_{2}=\sqrt{-q_{2}}\left(c_{1}^{*}\right)^{2}=\sqrt{q_{1} q_{2}} c_{1} c_{1}^{*}=\left(q_{1} / \sqrt{-q_{2}}\right) c_{1}^{2}=\sqrt{q_{1} / q_{2}} c_{1}^{*} c_{1}=0 .
$$

In particular we see that $c_{1}^{*} c_{1}=0$, and thus also $c_{1}=0$.

We note that since $C^{*}$-algebras are $*$-semisimple and thus very proper, Theorem 1.5 is a special case of Corollary 3.4 and Propositions 3.6 and 3.8.

3.9 Proposition. Let $c$ be an element of a *-semisimple Banach *-algebra $\mathscr{A}$, and let $q_{1} \in \mathbb{R} \backslash\{0, \pm 1\}$. Then the elements $c_{1}=c$ and $c_{2}=c c^{*}-q_{1} c^{*} c$ satisfy the $*$-algebraic $\left(q_{1},-q_{1}^{-1}\right)$-deformed Heisenberg-Lie commutation relations if and only if $c^{2}=0$.

Proof. By Lemma 3.5, we must show that $q_{1} c^{*} c^{2}-q_{1}^{-1} c^{2} c^{*}=0$ if and only if $c^{2}=0$. The implication $\Leftarrow$ is obvious. Conversely, suppose that $q_{1} c^{*} c^{2}-q_{1}^{-1} c^{2} c^{*}=0$. Left- and right-multiplying by $q_{1} c^{*}$ and rearranging yields $q_{1}^{2}\left(c^{*}\right)^{2} c^{2}=c^{*} c^{2} c^{*}$ and $q_{1}^{2} c^{*} c^{2} c^{*}=c^{2}\left(c^{*}\right)^{2}$. Substituting the first of these equations into the second gives $q_{1}^{4}\left(c^{*}\right)^{2} c^{2}=c^{2}\left(c^{*}\right)^{2}$. By $*$-semisimplicity, we can take a Hilbert space $H$ and an injective $*$-homomorphism $\varphi: \mathscr{A} \rightarrow \mathscr{B}(H)$, and we then have $q_{1}^{4}\left(\varphi(c)^{*}\right)^{2} \varphi(c)^{2}=\varphi(c)^{2}\left(\varphi(c)^{*}\right)^{2}$. It follows that $\left|q_{1}\right|^{4}\left\|\varphi(c)^{2}\right\|^{2}=\left\|\varphi(c)^{2}\right\|^{2}$, and thus $\left(1-\left|q_{1}\right|^{4}\right)\left\|\varphi(c)^{2}\right\|^{2}=0$. Now $\left|q_{1}\right|^{4} \neq 1$ because $q_{1} \neq \pm 1$, and therefore $0=\varphi(c)^{2}=\varphi\left(c^{2}\right)$. Hence $c^{2}=0$ by injectivity of $\varphi$.

3.10 Remark. Proposition 3.9 does not extend to the class of all Banach *-algebras. Indeed, for any $q_{1}, q_{2} \in \mathbb{R} \backslash\{0\}$, consider the Banach $*$-algebra $\mathscr{C}_{q_{1}, q_{2}}$ constructed in the proof of Theorem 1.4 and the elements $c_{1}, c_{2} \in \mathscr{C}_{q_{1}, q_{2}}$ satisfying the $*$-algebraic $\left(q_{1}, q_{2}\right)$-deformed Heisenberg-Lie commutation relations. By definition, $c_{1}=\pi\left(\delta_{s_{1}}\right)$, so that $c_{1}^{2}=\pi\left(\delta_{s_{1}^{2}}\right)$; consequently, as in the proof of Theorem 1.2, we obtain

$$
\begin{aligned}
1 & =\left\|\delta_{s_{1}^{2}}\right\|_{1} \geqslant\left\|\pi\left(\delta_{s_{1}^{2}}\right)\right\|=\inf \left\{\left\|\delta_{s_{1}^{2}}-g\right\|_{1} \mid g \in \mathscr{J}\right\} \\
& \geqslant \inf \left\{\left\|P_{2}\left(\delta_{s_{1}^{2}}-g\right)\right\|_{1} \mid g \in \mathscr{J}\right\}=\inf \left\{\left\|\delta_{s_{1}^{2}}\right\|_{1} \mid g \in \mathscr{J}\right\}=1,
\end{aligned}
$$

showing that $\left\|c_{1}^{2}\right\|=1$. In particular, $c_{1}^{2} \neq 0$, and Proposition 3.9 implies that $\mathscr{C}_{q_{1},-q_{1}^{-1}}$ is not $*$-semisimple for any $q_{1} \in \mathbb{R} \backslash\{0, \pm 1\}$.

3.11 Proposition. Let $c$ be a non-zero normal element of a proper $*$-algebra $\mathscr{A}$, and let $q_{1}, q_{2} \in \mathbb{R} \backslash\{0\}$. Then the elements $c_{1}=c$ and $c_{2}=c c^{*}-q_{1} c^{*} c$ satisfy the $*$-algebraic $\left(q_{1}, q_{2}\right)$-deformed Heisenberg-Lie commutation relations if and only if $q_{1}=1$ (in which case $\left.c_{2}=0\right)$ or $q_{2}=1$.

Proof. Since $c$ is normal, the identity in Lemma 3.5(a) reduces to

$$
\left(1+q_{1} q_{2}-q_{1}-q_{2}\right) c c^{*} c=0 .
$$

Now $c c^{*} c \neq 0$ because $c \neq 0$ and $\mathscr{A}$ is proper, so Lemma 3.5 implies that $c_{1}$ and $c_{2}$ satisfy the $*$-algebraic $\left(q_{1}, q_{2}\right)$-deformed Heisenberg-Lie commutation relations if and only if

$$
0=1+q_{1} q_{2}-q_{1}-q_{2}=\left(1-q_{1}\right)\left(1-q_{2}\right),
$$


that is, if and only if $q_{1}=1$ or $q_{2}=1$.

In the light of Propositions 3.3, 3.6, 3.8, and 3.9, it is interesting to know what $*$-algebraic properties the Banach $*$-algebras $\mathscr{C}_{q_{1}, q_{2}}$ defined in the proof of Theorem 1.4 possess.

3.12 Definition. A $*$-algebra $\mathscr{A}$ is hermitian if each self-adjoint element $a$ of $\mathscr{A}$ has real spectrum, that is, if $a-\alpha 1_{\mathscr{A} \sharp}$ is invertible in $\mathscr{A}^{\sharp}$ whenever $a=a^{*}$ and $\alpha \in \mathbb{C} \backslash \mathbb{R}$.

It is shown in $[4$, example $3.1 .4(\mathrm{iv})]$ that $\ell_{1}\left(\mathbb{S}_{2}\right)$ is not hermitian. We imitate the proof of this result to obtain the same conclusion for $\mathscr{C}_{q_{1}, q_{2}}$. For the convenience of the reader we include full details.

3.13 Proposition. For each pair $q_{1}, q_{2} \in \mathbb{R} \backslash\{0\}$, the Banach $*$-algebra $\mathscr{C}_{q_{1}, q_{2}}$ defined in the proof of Theorem 1.4 is not hermitian.

Proof. Given $\xi, \eta \in \overline{\mathbb{D}}$ (the closed unit ball in $\mathbb{C}$ ), Lemma 2.4 implies that there is a unique bounded unital algebra homomorphism $\theta_{\xi, \eta}: \ell_{1}\left(\mathbb{S}_{2}\right) \rightarrow \mathbb{C}$ such that $\theta_{\xi, \eta}\left(\delta_{s_{1}}\right)=\xi$ and $\theta_{\xi, \eta}\left(\delta_{s_{2}}\right)=\eta$. More explicitly, we have

$$
\theta_{\xi, \eta}(f)=\sum_{w \in \mathbb{S}_{2}} f(w) \xi^{n_{1}(w)} \eta^{n_{2}(w)} \quad\left(f \in \ell_{1}\left(\mathbb{S}_{2}\right)\right)
$$

where $n_{1}(w)$ and $n_{2}(w)$ denote the total number of times that $s_{1}$ and $s_{2}$, respectively, occur in $w \in \mathbb{S}_{2}$. In particular it follows that

$$
\theta_{\xi, \eta}\left(g_{1}\right)=\left(q_{2}-q_{1} q_{2}-1+q_{1}\right) \xi^{2} \eta \quad \text { and } \quad \theta_{\xi, \eta}\left(g_{2}\right)=\left(1-q_{1}-q_{2}+q_{1} q_{2}\right) \xi \eta^{2},
$$

where $g_{1}$ and $g_{2}$ are defined as in (2.4). Taking $\eta=0$, we see that $g_{1}, g_{2} \in \operatorname{ker} \theta_{\xi, 0}$, and so $\mathscr{J} \subseteq \operatorname{ker} \theta_{\xi, 0}$. Hence the first isomorphism theorem implies that there is a unique bounded unital algebra homomorphism $\psi_{\xi}: \mathscr{C}_{q_{1}, q_{2}} \rightarrow \mathbb{C}$ such that $\theta_{\xi, 0}=\psi_{\xi} \circ \pi$. With $c_{1}=\pi\left(\delta_{s_{1}}\right)$ (as in the proof of Theorem 1.4), we have

$$
\psi_{\xi}\left(c_{1}^{*}\right)=\psi_{\xi}\left(\pi\left(\delta_{s_{1}}\right)^{*}\right)=\psi_{\xi}\left(\pi\left(\delta_{s_{1}}^{*}\right)\right)=\theta_{\xi, 0}\left(\delta_{s_{2}}\right)=0 \quad \text { and } \quad \psi_{\xi}\left(c_{1}\right)=\theta_{\xi, 0}\left(\delta_{s_{1}}\right)=\xi,
$$

so that $\psi_{\xi}$ is not a $*$-homomorphism when $\xi \neq 0$. However, each multiplicative linear functional on a hermitian *-algebra is automatically a $*$-homomorphism by [4, proposition $1.10 .22(\mathrm{i})$ ], and consequently $\mathscr{C}_{q_{1}, q_{2}}$ cannot be hermitian.

3.14 Remark. It is not hard to display explicitly a self-adjoint element of $\mathscr{C}_{q_{1}, q_{2}}$ whose spectrum is not real. Indeed, set $h=\delta_{s_{1}}+\delta_{s_{2}} \in \ell_{1}\left(\mathbb{S}_{2}\right)$. Then $h$ is self-adjoint, and therefore $\pi(h) \in \mathscr{C}_{q_{1}, q_{2}}$ is self-adjoint. By elementary spectral theory (e.g., see [4, proposition 1.5.28]), we have

$$
\sigma(\pi(h)) \cup\{0\} \ni \psi_{\xi}(\pi(h))=\theta_{\xi, 0}(h)=\xi \quad(\xi \in \overline{\mathbb{D}}),
$$

so that $\overline{\mathbb{D}} \subseteq \sigma(\pi(h)) \cup\{0\}$. 


\section{$4 \quad$ Weighted shift operators satisfying the $*$-algebraic Heisenberg-Lie commutation relations}

In this section we investigate for which pairs $q_{1}, q_{2} \in \mathbb{R} \backslash\{0\}$ there is a bounded weight sequence $\omega$ such that the corresponding weighted right-shift operator $R_{\omega}$ on $\ell_{2}(\mathbb{Z})$ together with the operator $R_{\omega} R_{\omega}^{*}-q_{1} R_{\omega}^{*} R_{\omega}$ satisfy the $*$-algebraic $\left(q_{1}, q_{2}\right)$-deformed Heisenberg-Lie commutation relations.

We begin by making precise what we mean by a 'weighted right-shift operator'.

4.1 Construction. Let $\left(e_{n}\right)_{n \in \mathbb{Z}}$ denote the standard orthonormal basis for the Hilbert space $\ell_{2}(\mathbb{Z})$. For each $\omega=\left(\omega_{n}\right)_{n \in \mathbb{Z}} \in \ell_{\infty}(\mathbb{Z})$, the weighted right-shift operator

$$
R_{\omega}: \sum_{n \in \mathbb{Z}} \alpha_{n} e_{n} \mapsto \sum_{n \in \mathbb{Z}} \alpha_{n} \omega_{n} e_{n+1}, \quad \ell_{2}(\mathbb{Z}) \rightarrow \ell_{2}(\mathbb{Z})
$$

is bounded and linear, with norm $\left\|R_{\omega}\right\|=\|\omega\|_{\infty}$; its adjoint is the weighted left-shift operator given by

$$
R_{\omega}^{*}: \quad \sum_{n \in \mathbb{Z}} \alpha_{n} e_{n} \mapsto \sum_{n \in \mathbb{Z}} \alpha_{n} \bar{\omega}_{n-1} e_{n-1}, \quad \ell_{2}(\mathbb{Z}) \rightarrow \ell_{2}(\mathbb{Z}),
$$

as is easily verified.

4.2 Lemma. For each pair $q_{1}, q_{2} \in \mathbb{R} \backslash\{0\}$ and each weight $\omega=\left(\omega_{n}\right)_{n \in \mathbb{Z}} \in \ell_{\infty}(\mathbb{Z})$, the following three conditions are equivalent:

(a) for each $n \in \mathbb{Z}$, either $\omega_{n}=0$ or $q_{1}\left|\omega_{n+1}\right|^{2}+q_{2}\left|\omega_{n-1}\right|^{2}=\left(1+q_{1} q_{2}\right)\left|\omega_{n}\right|^{2}$;

(b) $q_{1} R_{\omega}^{*} R_{\omega}^{2}+q_{2} R_{\omega}^{2} R_{\omega}^{*}=\left(1+q_{1} q_{2}\right) R_{\omega} R_{\omega}^{*} R_{\omega}$;

(c) the operators $c_{1}=R_{\omega}$ and $c_{2}=R_{\omega} R_{\omega}^{*}-q_{1} R_{\omega}^{*} R_{\omega}$ satisfy the *-algebraic $\left(q_{1}, q_{2}\right)$ deformed Heisenberg-Lie commutation relations.

Proof. The equivalence of (b) and (c) is immediate from Lemma 3.5(a)-(b).

To prove that (a) and (b) are equivalent, we observe that, for each $n \in \mathbb{Z}$,

$$
\left(q_{1} R_{\omega}^{*} R_{\omega}^{2}+q_{2} R_{\omega}^{2} R_{\omega}^{*}\right) e_{n}=\left(q_{1}\left|\omega_{n+1}\right|^{2}+q_{2}\left|\omega_{n-1}\right|^{2}\right) \omega_{n} e_{n+1} \quad \text { and } R_{\omega} R_{\omega}^{*} R_{\omega} e_{n}=\left|\omega_{n}\right|^{2} \omega_{n} e_{n+1} \text {. }
$$

Hence (b) is satisfied if and only if

$$
\left(q_{1}\left|\omega_{n+1}\right|^{2}+q_{2}\left|\omega_{n-1}\right|^{2}\right) \omega_{n}=\left(1+q_{1} q_{2}\right)\left|\omega_{n}\right|^{2} \omega_{n} \quad(n \in \mathbb{Z}),
$$

which is clearly equivalent to (a).

4.3 Remark. Another three equivalent conditions can easily be added to Lemma 4.2 using the counterparts of Lemma 3.5(c)-(e). 
Guided by the condition in Lemma 4.2(a), we seek to characterize the sequences $\left(\rho_{n}\right)_{n \in \mathbb{Z}}=\left(\left|\omega_{n}\right|^{2}\right)_{n \in \mathbb{Z}}$ which satisfy

$$
\rho_{n}=0 \quad \text { or } \quad q_{1} \rho_{n+1}+q_{2} \rho_{n-1}=\left(1+q_{1} q_{2}\right) \rho_{n} \quad(n \in \mathbb{Z}) .
$$

We begin with the case where $q_{1} q_{2}=-1$. This case is simpler and requires special treatment because the right-hand side of the second equation in (4.1) vanishes, thus allowing us to reduce (4.1) to the simplified form (4.2) below.

4.4 Lemma. Let $q_{1} \in \mathbb{R} \backslash\{0\}$. A complex sequence $\left(\rho_{n}\right)_{n \in \mathbb{Z}}$ satisfies

$$
\rho_{n}=0 \quad \text { or } \quad q_{1}^{2} \rho_{n+1}=\rho_{n-1} \quad(n \in \mathbb{Z})
$$

if and only if one of the following two conditions is satisfied:

(i) for each $n \in \mathbb{Z}, \rho_{n} \neq 0$ implies that $\rho_{n-1}=\rho_{n+1}=0$; or

(ii) for each $n \in \mathbb{Z}, \rho_{2 n}=q_{1}^{-2 n} \rho_{0}$ and $\rho_{2 n+1}=q_{1}^{-2 n} \rho_{1}$.

Proof. It is easy to check that any sequence $\left(\rho_{n}\right)_{n \in \mathbb{Z}}$ satisfying either (i) or (ii) will also satisfy (4.2).

Conversely, suppose that $\left(\rho_{n}\right)_{n \in \mathbb{Z}}$ satisfies (4.2), but fails (i). Then there is an integer $N$ such that $\rho_{N} \neq 0$ and $\rho_{N+1} \neq 0$. Two applications of (4.2) show that $\rho_{N-1}=q_{1}^{2} \rho_{N+1}$ and $\rho_{N+2}=q_{1}^{-2} \rho_{N}$. In particular $\rho_{N-1} \neq 0$ and $\rho_{N+2} \neq 0$, so we may apply (4.2) again to obtain $\rho_{N-2}=q_{1}^{2} \rho_{N}$ and $\rho_{N+3}=q_{1}^{-2} \rho_{N+1}$. Continuing by induction leads to the conclusion that $\rho_{N+2 n}=q_{1}^{-2 n} \rho_{N}$ and $\rho_{N+2 n+1}=q_{1}^{-2 n} \rho_{N+1}$ for each $n \in \mathbb{Z}$; clearly this implies (ii).

4.5 Corollary. Let $q_{1} \in \mathbb{R} \backslash\{0\}$, and let $\omega=\left(\omega_{n}\right)_{n \in \mathbb{Z}} \in \ell_{\infty}(\mathbb{Z})$. Then the operators $c_{1}=R_{\omega}$ and $c_{2}=R_{\omega} R_{\omega}^{*}-q_{1} R_{\omega}^{*} R_{\omega}$ on $\ell_{2}(\mathbb{Z})$ satisfy the $*$-algebraic $\left(q_{1},-q_{1}^{-1}\right)$-deformed Heisenberg-Lie commutation relations if and only if one of the following two conditions is satisfied:

(i) for each $n \in \mathbb{Z}, \omega_{n} \neq 0$ implies that $\omega_{n-1}=\omega_{n+1}=0$; or

(ii) $q_{1}= \pm 1$ and for each $n \in \mathbb{Z},\left|\omega_{2 n}\right|=\left|\omega_{0}\right|$ and $\left|\omega_{2 n+1}\right|=\left|\omega_{1}\right|$.

Proof. By Lemmas 4.2 and $4.4, c_{1}$ and $c_{2}$ satisfy the $*$-algebraic $\left(q_{1},-q_{1}^{-1}\right)$-deformed Heisenberg-Lie commutation relations if and only if the sequence $\left(\rho_{n}\right)_{n \in \mathbb{Z}}=\left(\left|\omega_{n}\right|^{2}\right)_{n \in \mathbb{Z}}$ satisfies one of the following two conditions:

(i') for each $n \in \mathbb{Z},\left|\omega_{n}\right|^{2} \neq 0$ implies that $\left|\omega_{n-1}\right|^{2}=\left|\omega_{n+1}\right|^{2}=0$; or

(ii') for each $n \in \mathbb{Z},\left|\omega_{2 n}\right|^{2}=q_{1}^{-2 n}\left|\omega_{0}\right|^{2}$ and $\left|\omega_{2 n+1}\right|^{2}=q_{1}^{-2 n}\left|\omega_{1}\right|^{2}$.

Clearly conditions (i) and (i') are equivalent, and (ii) implies (ii').

Conversely, suppose that $\left(\mathrm{ii}^{\prime}\right)$ is satisfied. If $\omega_{0}=0$, then $\omega_{2 n}=0$ for each $n \in \mathbb{Z}$, so that (i) is satisfied. Otherwise we have $q_{1}^{-2 n}=\left|\omega_{2 n}\right|^{2} /\left|\omega_{0}\right|^{2}$ for each $n \in \mathbb{Z}$. As $\omega$ is bounded, this implies that the sequence $\left(q_{1}^{-2 n}\right)_{n \in \mathbb{Z}}$ is bounded, and consequently $q_{1}= \pm 1$. Hence (ii) is satisfied. 
4.6 Remark. Corollary 4.5(i) implies that $R_{\omega}^{2}=0$, in accordance with Proposition 3.9.

Having thus settled the case where $q_{1} q_{2}=-1$, we proceed to prove a counterpart of Lemma 4.4 for $q_{1} q_{2} \neq-1$. This requires the following concept.

4.7 Definition. For $n \in \mathbb{Z}$ and $q \in \mathbb{R} \backslash\{0\}$, the $n^{\text {th }} q$-number is given by

$$
\{n\}_{q}= \begin{cases}n & \text { if } q=1 \\ \frac{q^{n}-1}{q-1} & \text { otherwise. }\end{cases}
$$

We note that, for each $q \in \mathbb{R} \backslash\{0\}$, the $q$-numbers satisfy the recurrence relation

$$
\{0\}_{q}=0 \quad \text { and } \quad\{n+1\}_{q}=1+q\{n\}_{q} \quad(n \in \mathbb{Z}),
$$

and $\{n\}_{q}=\sum_{j=0}^{n-1} q^{j}$ for $n \in \mathbb{N}$.

4.8 Lemma. Let $q_{1}, q_{2} \in \mathbb{R} \backslash\{0\}$ with $q_{1} q_{2} \neq-1$. A complex sequence $\left(\rho_{n}\right)_{n \in \mathbb{Z}}$ satisfies (4.1) if and only if one of the following five conditions is satisfied:

(i) $\rho_{n}=0 \quad(n \in \mathbb{Z})$;

(ii) there is an integer $M$ such that $\rho_{n}=0$ if and only if $n>M$, and

$$
\rho_{M-n}=q_{2}^{-n}\{n+1\}_{q_{1} q_{2}} \rho_{M} \quad(n \in \mathbb{N})
$$

(iii) there is an integer $N$ such that $\rho_{n}=0$ if and only if $n<N$, and

$$
\rho_{N+n}=q_{1}^{-n}\{n+1\}_{q_{1} q_{2}} \rho_{N} \quad(n \in \mathbb{N})
$$

(iv) there are integers $M<N$ such that $\rho_{n}=0$ if and only if $M<n<N$, and $\rho_{M-n}$ and $\rho_{N+n}$ are given by (4.3) and (4.4), respectively, for each $n \in \mathbb{N}$;

(v) $\rho_{n}=q_{1}^{-n}\left(\rho_{0}+\left(q_{1} \rho_{1}-\rho_{0}\right)\{n\}_{q_{1} q_{2}}\right) \neq 0$ for each $n \in \mathbb{Z}$.

Proof. It is straightforward to check that a sequence which satisfies one of the conditions (i)-(v) will also satisfy (4.1).

Conversely, suppose that the sequence $\left(\rho_{n}\right)_{n \in \mathbb{Z}}$ satisfies (4.1). If $\rho_{n}=0$ for each $n \in \mathbb{Z}$, then (i) is satisfied. On the other hand, if $\rho_{n} \neq 0$ for each $n \in \mathbb{Z}$, then an inductive argument based on the second equation in (4.1) shows that (v) is satisfied. Thus it remains to deal with the case where $\rho_{n}=0$ for some, but not all, $n \in \mathbb{Z}$. There are three possible scenarios to consider.

First, if there is an integer $M$ such that $\rho_{n}=0$ for each $n>M$, then by choosing $M$ minimal (that is, such that $\rho_{M} \neq 0$ ), an inductive argument based on (4.1) shows that $\rho_{M-n}$ is non-zero and given by (4.3) for each $n \in \mathbb{N}$, and therefore (ii) is satisfied.

Second, if there is an integer $N$ such that $\rho_{n}=0$ for each $n<N$, then an argument similar to the one just outlined establishes that we are in case (iii). 
Third, if none of the above applies, then there are integers $M<N$ such that $\rho_{M} \neq 0$, $\rho_{n}=0$ for $M<n<N$, and $\rho_{N} \neq 0$. Repeating the inductive arguments used to establish cases (ii) and (iii) above, we see that for each $n \in \mathbb{N}, \rho_{M-n}$ and $\rho_{N+n}$ are non-zero and given by (4.3) and (4.4), respectively, and so (iv) is satisfied.

4.9 Lemma. Let $r, s \in \mathbb{R} \backslash\{0\}$ with $r s \neq \pm 1$, and let $u, v \in \mathbb{R}$. Then the sequence $\left(u r^{n}+v s^{-n}\right)_{n \in \mathbb{N}}$ is bounded if and only if $(u=0$ or $|r| \leqslant 1)$ and $(v=0$ or $|s| \geqslant 1)$.

Proof. The implication ' $\Leftarrow$ ' is easy because if $u=0$ or $|r| \leqslant 1$, then the sequence $\left(u r^{n}\right)_{n \in \mathbb{N}}$ is bounded, and if $v=0$ or $|s| \geqslant 1$, then the sequence $\left(v s^{-n}\right)_{n \in \mathbb{N}}$ is bounded.

We prove the converse by contraposition. Suppose that $u \neq 0$ and $|r|>1$, or that $v \neq 0$ and $|s|<1$, and consider the following four cases:

(i) If $u=0$, then $v \neq 0$ and $|s|<1$, and so $\left|u r^{n}+v s^{-n}\right|=|v||s|^{-n} \rightarrow \infty$ as $n \rightarrow \infty$.

(ii) Similarly, if $v=0$, then $u \neq 0$ and $|r|>1$, so that $\left|u r^{n}+v s^{-n}\right|=|u||r|^{n} \rightarrow \infty$ as $n \rightarrow \infty$.

(iii) If $|r s|>1$, then also $|r|>1$ (because either $|r|>1$ or $|s|<1$, and in the latter case we have $|r|=|r s| /|s|>1$ ); by (i), we may suppose that $u \neq 0$, and it then follows that

$$
\left|u r^{n}+v s^{-n}\right| \geqslant|u||r|^{n}-|v||s|^{-n}=|r|^{n}\left(|u|-|v||r s|^{-n}\right) \rightarrow \infty \quad \text { as } \quad n \rightarrow \infty .
$$

(iv) Finally, if $|r s|<1$, then necessarily $|s|<1$, and so, assuming in addition that $v \neq 0$ (as we may by (ii)), we obtain

$$
\left|u r^{n}+v s^{-n}\right| \geqslant|v||s|^{-n}-|u||r|^{n}=|s|^{-n}\left(|v|-|u||r s|^{n}\right) \rightarrow \infty \quad \text { as } \quad n \rightarrow \infty .
$$

Thus in each case the sequence $\left(u r^{n}+v s^{-n}\right)_{n \in \mathbb{N}}$ is unbounded, as required.

4.10 Lemma. Let $r, s \in \mathbb{R} \backslash\{0\}$ with $r s \neq-1$, and define $t_{n}=s^{-n}\{n+1\}_{r s} \in \mathbb{R}$ for each $n \in \mathbb{N}$. Then:

(i) $t_{n}>0$ for each $n \in \mathbb{N}$ if and only if $r>-s^{-1}$;

(ii) $\left(t_{n}\right)_{n \in \mathbb{N}}$ is bounded if and only if $|r| \leqslant 1,|s| \geqslant 1$, and $(r, s) \neq \pm(1,1)$.

Proof. (i). The implication ' $\Rightarrow$ ' follows from the fact that $t_{1}=s^{-1}+r$ by definition, so that $t_{1}$ is positive if and only if $r>-s^{-1}$.

Conversely, suppose that $r>-s^{-1}$, and let $n \in \mathbb{N}$. First we consider the case where $s>0$. Then $r s>-1$, and we split in two further cases: if $r s>0$, then both factors $s^{-n}$ and $\{n+1\}_{r s}$ of $t_{n}$ are positive, so that $t_{n}$ is positive. Otherwise $0>r s>-1$, and we have

$$
t_{n}=\frac{(r s)^{n+1}-1}{s^{n}(r s-1)}
$$

by definition. Both the numerator and the denominator of this fraction are negative, and therefore $t_{n}$ is positive. 
Second, in the case where $s<0$, we have $r s<-1$, so that $t_{n}$ is given by (4.5). Now the numerator is positive for $n$ odd and negative for $n$ even, and the same is true for the denominator; consequently $t_{n}$ is always positive.

(ii). If $r s=1$, then $t_{n}=r^{n}(n+1)$, which is uniformly bounded in $n \in \mathbb{N}$ if and only if $|r|<1$. Since $s=r^{-1}$, this is equivalent to saying that $|r| \leqslant 1,|s| \geqslant 1$, and $(r, s) \neq \pm(1,1)$.

Otherwise $r s \neq 1$, and $t_{n}$ is given by (4.5), which can be rewritten as

$$
t_{n}=\frac{r s}{r s-1} r^{n}-\frac{1}{r s-1} s^{-n} \quad(n \in \mathbb{N}),
$$

showing that Lemma 4.9 applies with $u=r s /(r s-1)$ and $v=-1 /(r s-1)$. Since $u$ and $v$ are both non-zero, it follows that $\left(t_{n}\right)_{n \in \mathbb{N}}$ is bounded if and only if $|r| \leqslant 1$ and $|s| \geqslant 1$; the condition $(r, s) \neq \pm(1,1)$ is automatically satisfied because $r s \neq 1$.

4.11 Theorem. Let $q_{1}, q_{2} \in \mathbb{R} \backslash\{0\}$ with $q_{1} q_{2} \neq-1$, and let $\omega=\left(\omega_{n}\right)_{n \in \mathbb{Z}}$ be a complex sequence. Then $\omega$ is bounded and the operators $c_{1}=R_{\omega}$ and $c_{2}=R_{\omega} R_{\omega}^{*}-q_{1} R_{\omega}^{*} R_{\omega}$ on $\ell_{2}(\mathbb{Z})$ satisfy the $*$-algebraic $\left(q_{1}, q_{2}\right)$-deformed Heisenberg-Lie commutation relations if and only if one of the following four conditions is satisfied:

(i) $\omega_{n}=0 \quad(n \in \mathbb{Z})$;

(ii) $q_{1}$ and $q_{2}$ satisfy one of the following two conditions:

- $q_{1} \in(-1,0)$ and $q_{2} \in\left[1,-q_{1}^{-1}\right)$, or

- $q_{1} \in(0,1], q_{2} \in\left(-\infty,-q_{1}^{-1}\right) \cup[1, \infty)$, and $\left(q_{1}, q_{2}\right) \neq(1,1)$,

and there is an integer $M$ such that

$$
\omega_{n}=0 \quad(n>M), \quad \omega_{M} \neq 0, \quad \text { and } \quad\left|\omega_{M-n}\right|=\sqrt{q_{2}^{-n}\{n+1\}_{q_{1} q_{2}}}\left|\omega_{M}\right| \quad(n \in \mathbb{N}) ;
$$

(iii) $q_{1} \in(-\infty,-1) \cup[1, \infty)$ and $q_{2} \in\left(-q_{1}^{-1}, 1\right]$ with $\left(q_{1}, q_{2}\right) \neq(1,1)$, and there is an integer $N$ such that

$$
\omega_{n}=0 \quad(n<N), \quad \omega_{N} \neq 0, \quad \text { and } \quad\left|\omega_{N+n}\right|=\sqrt{q_{1}^{-n}\{n+1\}_{q_{1} q_{2}}}\left|\omega_{N}\right| \quad(n \in \mathbb{N})
$$

(iv) either $q_{1}=1$ or $q_{2}=1$ (or both), and $\left|\omega_{n}\right|=\left|\omega_{0}\right| \neq 0 \quad(n \in \mathbb{Z})$.

Proof. Lemma 4.10, together with the conditions imposed on $q_{1}$ and $q_{2}$ in (ii) and (iii), respectively, ensures that the arguments of the square roots in (ii) and (iii) are always positive and uniformly bounded in $n \in \mathbb{N}$; thus $\omega \in \ell_{\infty}(\mathbb{Z})$ whenever one of the conditions (i)-(iv) is satisfied, and we may therefore suppose that $\omega \in \ell_{\infty}(\mathbb{Z})$. Lemmas 4.2 and 4.8 then imply that the operators $c_{1}=R_{\omega}$ and $c_{2}=R_{\omega} R_{\omega}^{*}-q_{1} R_{\omega}^{*} R_{\omega}$ satisfy the $*$-algebraic $\left(q_{1}, q_{2}\right)$-deformed Heisenberg-Lie commutation relations if and only if the sequence $\left(\rho_{n}\right)_{n \in \mathbb{Z}}=\left(\left|\omega_{n}\right|^{2}\right)_{n \in \mathbb{Z}}$ satisfies one of the following five conditions:

(i') $\left|\omega_{n}\right|^{2}=0 \quad(n \in \mathbb{Z})$; 
(ii') there is an integer $M$ such that $\left|\omega_{n}\right|^{2}=0$ if and only if $n>M$, and

$$
\left|\omega_{M-n}\right|^{2}=q_{2}^{-n}\{n+1\}_{q_{1} q_{2}}\left|\omega_{M}\right|^{2} \quad(n \in \mathbb{N})
$$

(iii') there is an integer $N$ such that $\left|\omega_{n}\right|^{2}=0$ if and only if $n<N$, and

$$
\left|\omega_{N+n}\right|^{2}=q_{1}^{-n}\{n+1\}_{q_{1} q_{2}}\left|\omega_{N}\right|^{2} \quad(n \in \mathbb{N})
$$

$\left(\right.$ iv $\left.^{\prime}\right)$ there are integers $M<N$ such that $\left|\omega_{n}\right|^{2}=0$ if and only if $M<n<N$, and $\left|\omega_{M-n}\right|^{2}$ and $\left|\omega_{N+n}\right|^{2}$ are given by (4.6) and (4.7), respectively, for each $n \in \mathbb{N}$;

$\left(\mathrm{v}^{\prime}\right)\left|\omega_{n}\right|^{2}=q_{1}^{-n}\left(\left|\omega_{0}\right|^{2}+\left(q_{1}\left|\omega_{1}\right|^{2}-\left|\omega_{0}\right|^{2}\right)\{n\}_{q_{1} q_{2}}\right) \neq 0$ for each $n \in \mathbb{Z}$.

It remains to prove that one of the conditions (i)-(iv) is satisfied if and only if one of the conditions $\left(\mathrm{i}^{\prime}\right)-\left(\mathrm{v}^{\prime}\right)$ is satisfied.

Clearly (i) and (i') are equivalent.

We claim that (ii) and (ii') are equivalent. The implication (ii) $\Rightarrow\left(\right.$ ii $\left.^{\prime}\right)$ is obvious. Conversely, suppose that (ii') is satisfied. Then $q_{2}^{-n}\{n+1\}_{q_{1} q_{2}}=\left|\omega_{M-n}\right|^{2}\left|\omega_{M}\right|^{-2}$ for each $n \in \mathbb{N}$; as the expression on the right-hand side is positive and uniformly bounded in $n$, the same is true for the left-hand side. Hence Lemma 4.10 implies that $q_{1}>-q_{2}^{-1},\left|q_{1}\right| \leqslant 1,\left|q_{2}\right| \geqslant 1$, and $\left(q_{1}, q_{2}\right) \neq \pm(1,1)$. We now split in two cases:

- If $q_{2} \leqslant-1$, then $q_{1}>-q_{2}^{-1}$ implies that $q_{1} q_{2}<-1$, so that $q_{1}>0$ and $q_{2}<-q_{1}^{-1}$; combining this with the fact that $\left|q_{1}\right| \leqslant 1$ gives $q_{1} \in(0,1]$ and $q_{2} \in\left(-\infty,-q_{1}^{-1}\right)$.

- Otherwise $q_{2} \geqslant 1$, and $q_{1}>-q_{2}^{-1}$ implies that $q_{1} q_{2}>-1$. If $q_{1}<0$, this means that $q_{2}<-q_{1}^{-1}$, so that $q_{1} \in(-1,0)$ and $q_{2} \in\left[1,-q_{1}^{-1}\right)$. (Note that we cannot have $q_{1}=-1$ because this would imply that $q_{1} q_{2}=-q_{2} \leqslant-1$.) Otherwise $q_{1}>0$, in which case $q_{1} q_{2}>-1$ is trivially satisfied, so that we have $q_{1} \in(0,1]$ and $q_{2} \in[1, \infty)$.

Hence $q_{1}$ and $q_{2}$ satisfy the conditions stated in (ii), and the formula for $\omega$ is immediate from (4.6).

A similar argument shows that (iii) and (iii') are equivalent.

Next, we prove that (iv) and $\left(\mathrm{v}^{\prime}\right)$ are equivalent. A straightforward calculation shows that (iv) implies $\left(\mathrm{v}^{\prime}\right)$. Conversely, suppose that $\left(\mathrm{v}^{\prime}\right)$ is satisfied. If $q_{1}\left|\omega_{1}\right|^{2}-\left|\omega_{0}\right|^{2}=0$, then $\left(\mathrm{v}^{\prime}\right)$ reduces to $\left|\omega_{n}\right|^{2}=q_{1}^{-n}\left|\omega_{0}\right|^{2} \neq 0$ for each $n \in \mathbb{Z}$, so that $\omega_{0} \neq 0$ and $q_{1}^{-n}=\left|\omega_{n}\right|^{2}\left|\omega_{0}\right|^{-2}$ for each $n \in \mathbb{Z}$. Since the right-hand side of this identity is positive and uniformly bounded in $n$, the same is true for the left-hand side, and therefore $q_{1}=1$. Hence (iv) is satisfied in this case.

Otherwise we have $q_{1}\left|\omega_{1}\right|^{2}-\left|\omega_{0}\right|^{2} \neq 0$. If $q_{1} q_{2}=1$, then by choosing $n \in \mathbb{Z}$ of suitably large absolute value and of suitable sign and parity, we can arrange that

$$
0>q_{1}^{-n}\left(\left|\omega_{0}\right|^{2}+\left(q_{1}\left|\omega_{1}\right|^{2}-\left|\omega_{0}\right|^{2}\right) n\right) .
$$


This, however, contradicts that the expression on the right-hand side is equal to $\left|\omega_{n}\right|^{2}>0$ by $\left(\mathrm{v}^{\prime}\right)$. Hence we must have $q_{1} q_{2} \neq 1$, and $\left(\mathrm{v}^{\prime}\right)$ can be rewritten as follows:

$$
\begin{aligned}
\left|\omega_{n}\right|^{2} & =q_{1}^{-n}\left(\left|\omega_{0}\right|^{2}+\left(q_{1}\left|\omega_{1}\right|^{2}-\left|\omega_{0}\right|^{2}\right) \frac{\left(q_{1} q_{2}\right)^{n}-1}{q_{1} q_{2}-1}\right) \\
& =\frac{q_{1}\left|\omega_{1}\right|^{2}-\left|\omega_{0}\right|^{2}}{q_{1} q_{2}-1} q_{2}^{n}+\frac{q_{1}\left(q_{2}\left|\omega_{0}\right|^{2}-\left|\omega_{1}\right|^{2}\right)}{q_{1} q_{2}-1} q_{1}^{-n}=u q_{2}^{n}+v q_{1}^{-n} \quad(n \in \mathbb{Z}),
\end{aligned}
$$

where we have introduced

$$
u=\frac{q_{1}\left|\omega_{1}\right|^{2}-\left|\omega_{0}\right|^{2}}{q_{1} q_{2}-1} \quad \text { and } \quad v=\frac{q_{1}\left(q_{2}\left|\omega_{0}\right|^{2}-\left|\omega_{1}\right|^{2}\right)}{q_{1} q_{2}-1} ;
$$

note that $u \neq 0$ by assumption. Boundedness of $\omega$ implies that both of the sequences $\left(u q_{2}^{n}+v q_{1}^{-n}\right)_{n \in \mathbb{N}}$ and $\left(u q_{2}^{-n}+v q_{1}^{n}\right)_{n \in \mathbb{N}}$ are bounded, and therefore $\left|q_{2}\right|=1$ and either $v=0$ or $\left|q_{1}\right|=1$ by Lemma 4.9. However, as $q_{1} q_{2} \neq \pm 1$, we cannot have $\left|q_{1}\right|=1$, so $v=0$, and consequently $q_{2}\left|\omega_{0}\right|^{2}=\left|\omega_{1}\right|^{2}$. It follows that $q_{2}=\left|\omega_{1}\right|^{2}\left|\omega_{0}\right|^{-2}>0$, so that $q_{2}=1$ and $\left|\omega_{0}\right|=\left|\omega_{1}\right|$. Substituting this in (4.8) shows that $\left|\omega_{n}\right|^{2}=u=\left|\omega_{0}\right|^{2}$ for each $n \in \mathbb{Z}$, so that (iv) is satisfied. Hence (iv) and $\left(\mathrm{v}^{\prime}\right)$ are equivalent.

Finally, we claim that no bounded sequence $\omega$ satisfies (iv'). Indeed, by Lemma 4.10(ii), boundedness of the sequences $\left(\left|\omega_{M-n}\right|^{2}\right)_{n \in \mathbb{N}}$ and $\left(\left|\omega_{N+n}\right|^{2}\right)_{n \in \mathbb{N}}$ given by (4.6) and (4.7), respectively, implies that $\left|q_{1}\right|=\left|q_{2}\right|=1$ and $\left(q_{1}, q_{2}\right) \neq \pm(1,1)$, so that $\left(q_{1}, q_{2}\right)= \pm(1,-1)$, contradicting our assumption that $q_{1} q_{2} \neq-1$.

4.12 Remark. (i) The conditions imposed on $q_{1}$ and $q_{2}$ in Theorem 4.11(ii)-(iv) ensure that there is no contradiction with Theorem 1.5(i) in the sense that if $q_{1}$ and $q_{2}$ satisfy one of the conditions (ii)-(iv) in Theorem 4.11, then they fail all five conditions in Theorem 1.5(i).

(ii) The operator $R_{\omega}$ is normal if and only if $\left|\omega_{n}\right|=\left|\omega_{0}\right|$ for each $n \in \mathbb{Z}$. Thus the conditions on $q_{1}$ and $q_{2}$ in Corollary 4.5(ii) and Theorem 4.11(iv) are in accordance with Proposition 3.11 and Theorem 1.5(ii).

4.13 Remark. Methods similar to those applied in this section can be used to construct (one-sided) weighted shift operators satisfying the $\left(q_{1}, q_{2}\right)$-deformed Heisenberg-Lie commutation relations on Banach spaces other than Hilbert space. Indeed, suppose that $E$ is a Banach space having a normalized unconditional basis $\left(e_{n}\right)_{n=1}^{\infty}$ which is equivalent to $\left(e_{n}\right)_{n=2}^{\infty}$. (Details of the unexplained terminology can be found in any standard text on Banach space theory, such as [9].) Then there are weighted right- and left-shift operators on $E$, and in analogy with the results above, one can characterize the weight sequences whose associated shift operators satisfy the $\left(q_{1}, q_{2}\right)$-deformed Heisenberg-Lie commutation relations. 


\section{Acknowledgements}

N. J. Laustsen was supported by the Danish Natural Science Research Council when this research was initiated.

S. D. Silvestrov was partially supported by the Crafoord Foundation, the Swedish Foundation of International Cooperation in Research and Higher Education (STINT), the Royal Physiographic Society in Lund, the Royal Swedish Academy of Sciences, the Swedish Research Council, and the Faculty of Engineering at Lund University.

\section{References}

[1] Yu. A. Bahturin, A. A. Mikhalev, V. M. Petrogradsky and M. V. Zaicev, Infinite dimensional Lie superalgebras, Walter de Gruyter, Berlin, 1992.

[2] C.-W. Chen, S. D. Silvestrov and F. Van Oystaeyen, Representations and cocycle twists of color Lie algebras, Algebras and Representation Theory 9 (2006), 633-650.

[3] C.-W. Chen, T. Petit and F. Van Oystaeyen, Note on cohomology of color Hopf and Lie algebras, J. Algebra 299 (2006), 419-442.

[4] H. G. Dales, Banach algebras and automatic continuity, London Mathematical Society Monographs 24, Clarendon Press, Oxford, 2000.

[5] M. F. Gorodnii and G. B. Podkolzin, Irreducible representations of a graded Lie algebra, in Spectral theory of operators and infinite-dimensional analysis, Institute of Mathematics, Academy of Sciences of Ukraine, Kiev, (1984), 66-77.

[6] V. A. Groza, I. I. Kachurik and A. U. Klimyk, $q$-Deformed Euclidean algebras and their representations, Teoret. Matem. Fiz. 103 (1995), 467-475.

[7] P. R. Halmos, A Hilbert space problem book (2 ${ }^{\text {nd }}$ edn), Graduate Texts in Mathematics 19, Springer-Verlag, Berlin, 1982.

[8] A. U. Klimyk, Nonstandard $q$-deformation of the Euclidean Lie algebra and its representations, Czechoslovak J. Phys. 48 (1998), 1395-1400.

[9] J. Lindenstrauss and L. Tzafriri, Classical Banach spaces, Vol. I, Ergebnisse der Mathematik und ihre Grenzgebiete 92, Springer-Verlag, Berlin, 1977.

[10] J. Lukierski and V. Rittenberg, Color-de Sitter and color-conformal superalgebras, Phys. Rev. D 18 (1978), 385-389.

[11] V. L. Ostrovskii and S. D. Silvestrov, Representations of the real forms of the graded analogue of the Lie algebra sl(2, C), Ukrain. Mat. Zh. 44 (1992), 1518-1524. (English translation: Ukrain. Math. J. 44 (1993), 1395-1401).) 
[12] L. Persson, S. D. Silvestrov and P. Strunk, Central elements of the second order in three-dimensional generalised Lie algebras, Czechoslovak J. Phys. 47 (1997), 99-106.

[13] D. Piontkovski and S. D. Silvestrov, Cohomology of 3-dimensional color Lie algebras, J. Algebra 316 (2007), 499-513.

[14] K. L. Price, Homological properties of color Lie superalgebras, Advances in Ring Theory (Granville, OH, 1996), Trends Math., Birkhäuser, Boston, MA, 1997, 287293.

[15] K. L. Price, Primeness criteria for universal enveloping algebras of Lie color algebras, J. Algebra 235 (2001), 589-607.

[16] C. R. Putnam, Commutation properties of Hilbert space operators and related topics, Springer-Verlag, Berlin, 1967.

[17] V. Rittenberg and D. Wyler, Generalized Superalgebras, Nucl. Phys. B139 (1978), 189-202.

[18] S. Sakai, Operator algebras in dynamical systems: the theory of unbounded derivations in $C^{*}$-algebras, Encyclopedia of mathematics and applications, Cambridge University Press, 1991.

[19] Yu. S. Samoilenko, Spectral theory of families of selfadjoint operators, Naukova Dumka, Kiev, 1984. (Extended English translation: Kluwer Academic Publ., Dordrecht, 1990.)

[20] M. Scheunert, Generalized Lie algebras, J. Math. Phys. 20 (1979), 712-720.

[21] M. Scheunert, Graded tensor calculus, J. Math. Phys. 24 (1983), 2658-2670.

[22] G. Sigurdsson and S. D. Silvestrov, Canonical involutions in three-dimensional generalised Lie algebras, Czechoslovak J. Phys. 50 (2000), 181-186.

[23] G. Sigurdsson and S. D. Silvestrov, Bosonic realizations of the colour Heisenberg-Lie algebra, J. Nonlinear Mathematical Physics 13 (2006), 110-128.

[24] S. D. Silvestrov, Hilbert space representations of the graded analogue of the Lie algebra of the group of plane motions, Studia Math. 117 (1996), 195-203.

[25] S. D. Silvestrov, On the classification of 3-dimensional coloured Lie algebras, in Quantum groups and quantum spaces, Banach Center Publications 40 (1997), 159-170.

[26] S. D. Silvestrov and L. B. Turowska, Representations of the $q$-deformed Lie algebra of the group of motions of the Euclidean plane, J. Functional Analysis 160 (1998), 79-114.

[27] H. Wielandt, Über die Unbeschränkheit der Schrödingerschen Operatoren der Quanten-mechanik, Math. Ann. 121 (1949), 21. 
[28] A. Wintner, The unboundedness of quantum-mechanical matrices, Phys. Rev. 71 (1947), 738-739.

Niels Jakob Laustsen,

Department of Mathematics and

Statistics,

Fylde College,

Lancaster University,

Lancaster LA1 4YF, England;

e-mail: n.laustsen@lancaster.ac.uk
Sergei D. Silvestrov,

Centre for Mathematical Sciences,

Division of Mathematics,

Faculty of Engineering (LTH),

Lund University, P.O. Box 118,

SE-221 00 Lund, Sweden;

e-mail: sergei.silvestrov@math.lth.se 